\title{
Functionalization of cyclodextrins via reactions of 2,3-anhydro-cyclodextrins
}

\author{
De-Qi Yuan, ${ }^{*[a]}$ Tsutomu Tahara, ${ }^{[b]}$ Wen-Hua Chen, ${ }^{[a]}$ Yuji Okabe, ${ }^{[a]}$ Cheng Yang, ${ }^{[a]}$ Youichi Yagi, ${ }^{[a]}$ Yasuyoshi Nogami, ${ }^{[b]}$ Makoto \\ Fukudome, ${ }^{[a]}$ Kahee Fujita ${ }^{[a]}$
}

[a] Department of Molecular Medicinal Sciences, Graduate School of Biomedical Sciences, Nagasaki University, Nagasaki 852-8521, Japan; [b] Daiichi College of Pharmaceutical Sciences, Tamagawa-cho, Minami-ku, Fukuoka 815-8511, Japan

\section{${ }^{1} \mathrm{H}$ and ${ }^{13} \mathrm{C}$ NMR spectra}

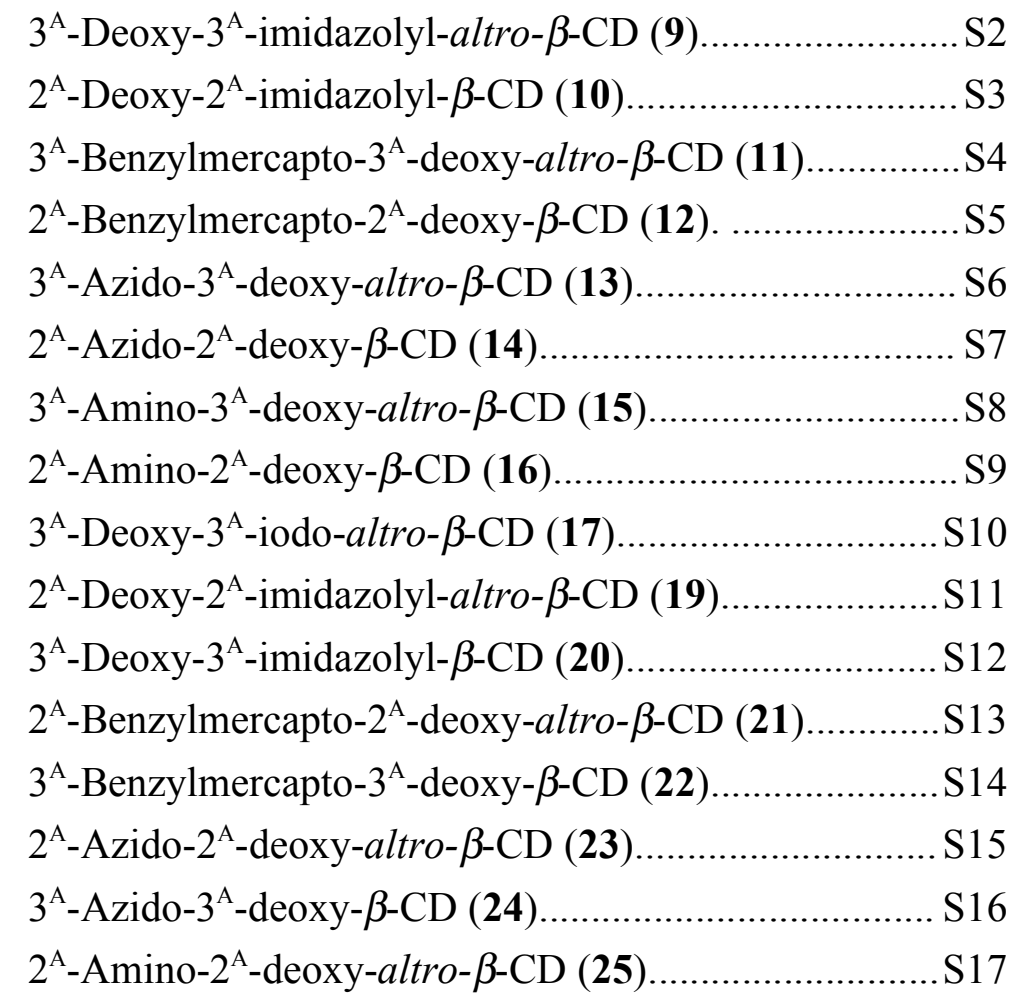

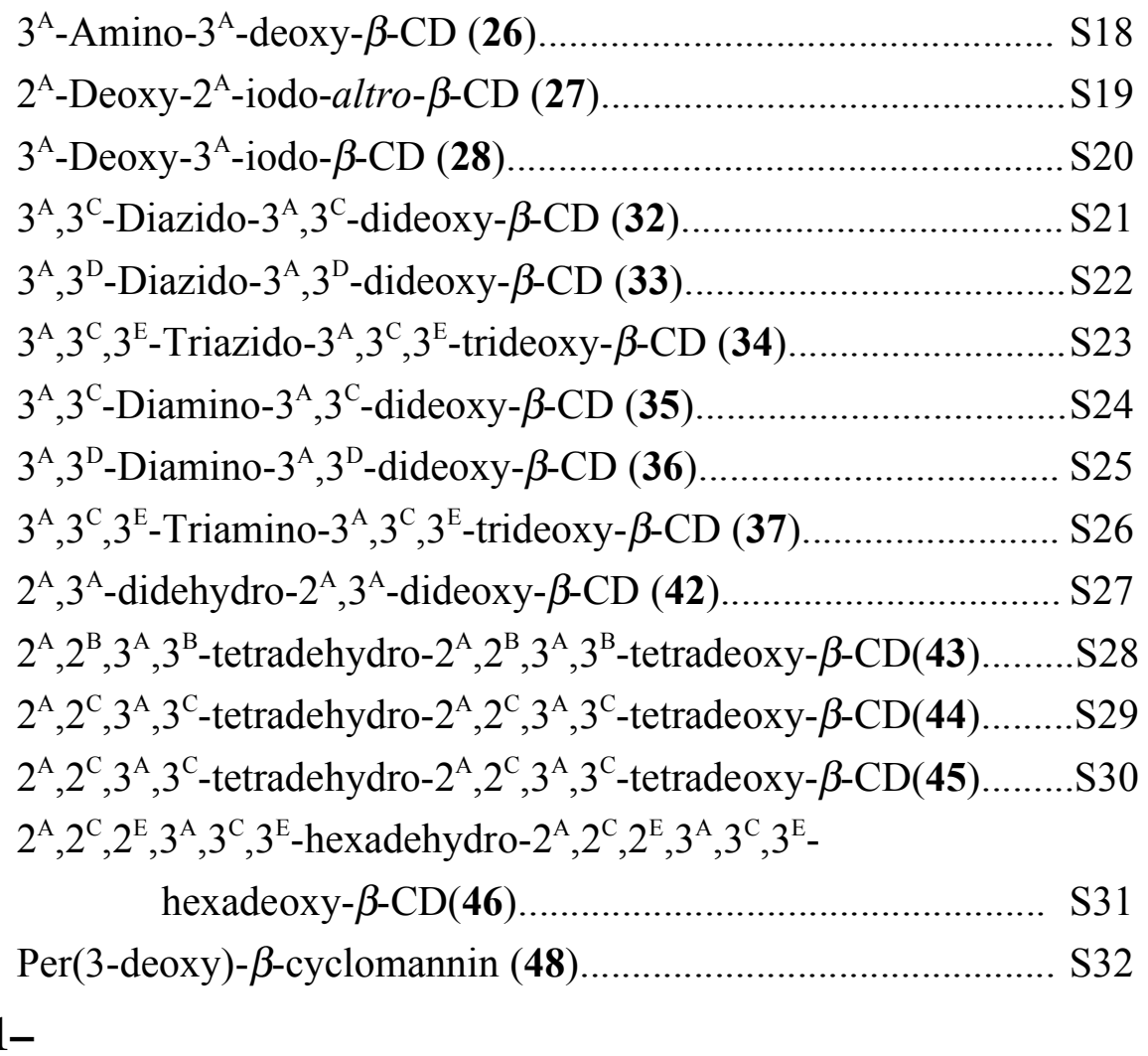



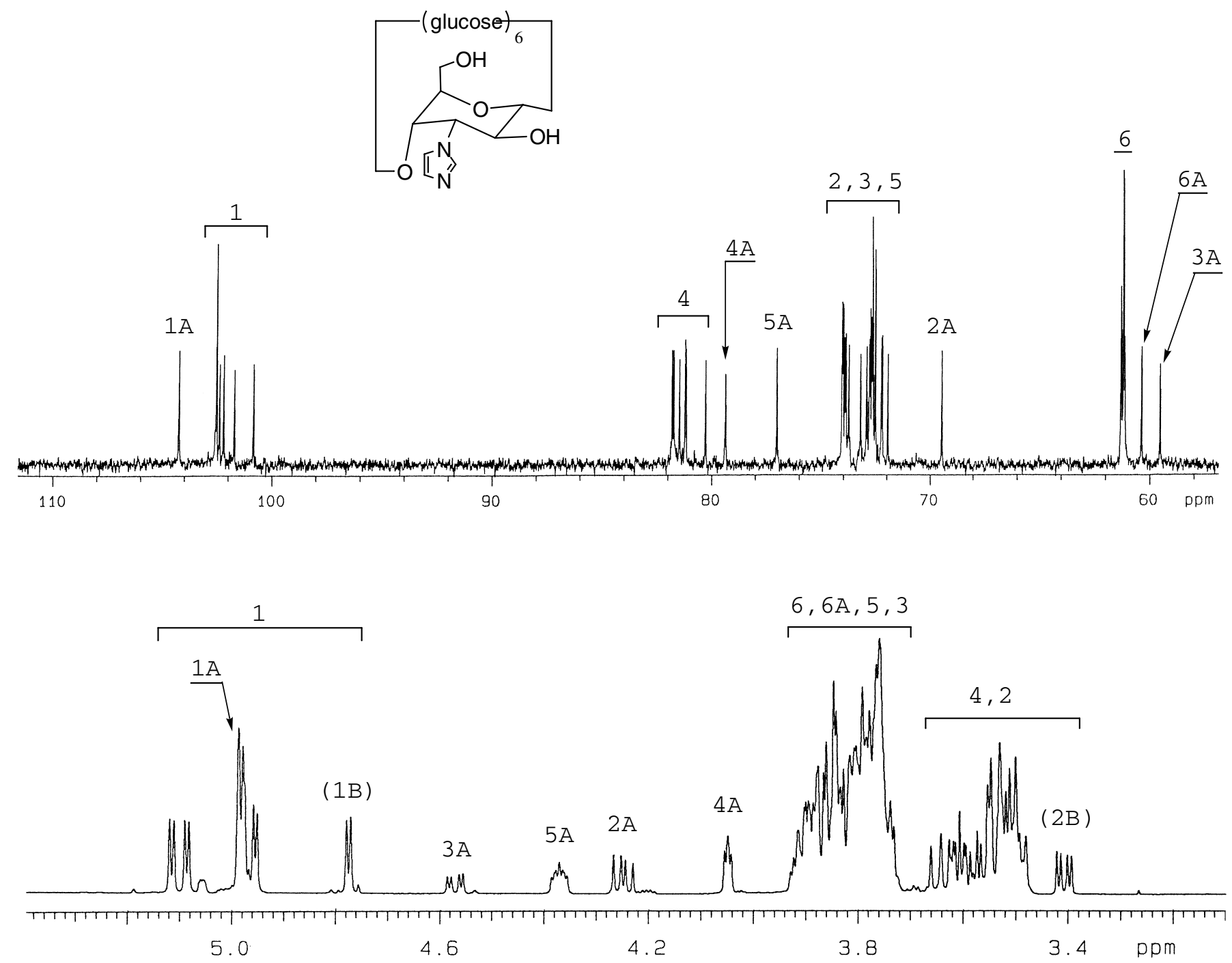

Fig. S2 ${ }^{1} \mathrm{H}(500 \mathrm{MHz})$ and ${ }^{13} \mathrm{C}$ NMR spectra $(125 \mathrm{MHz})$ of $3^{\mathrm{A}}$-deoxy- $3^{\mathrm{A}}$-imidazolyl-altro- $\beta$-CD 9 in $\mathrm{D}_{2} \mathrm{O}\left(\mathrm{CH}_{3} \mathrm{CN}\right.$ int $)$. 


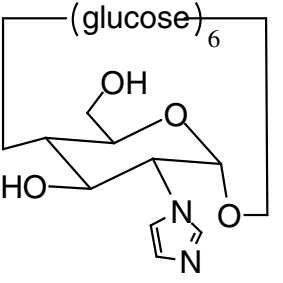

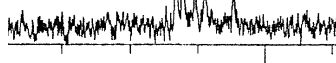

100

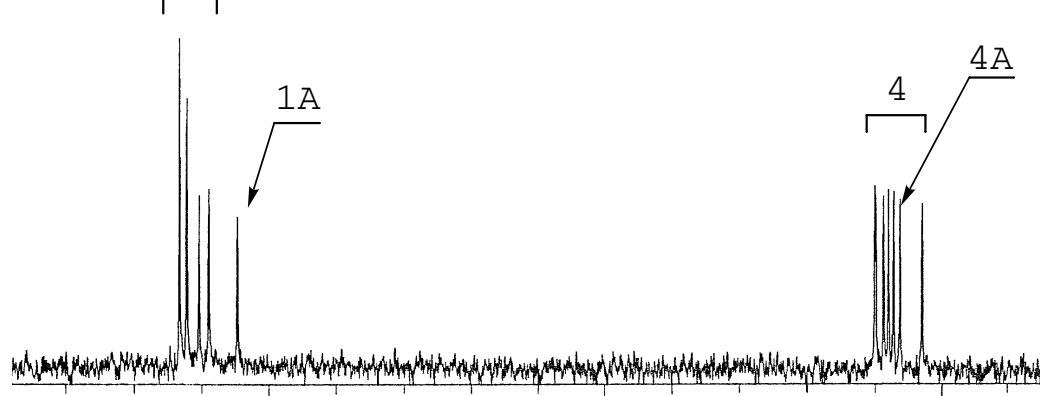

$2,3,5$

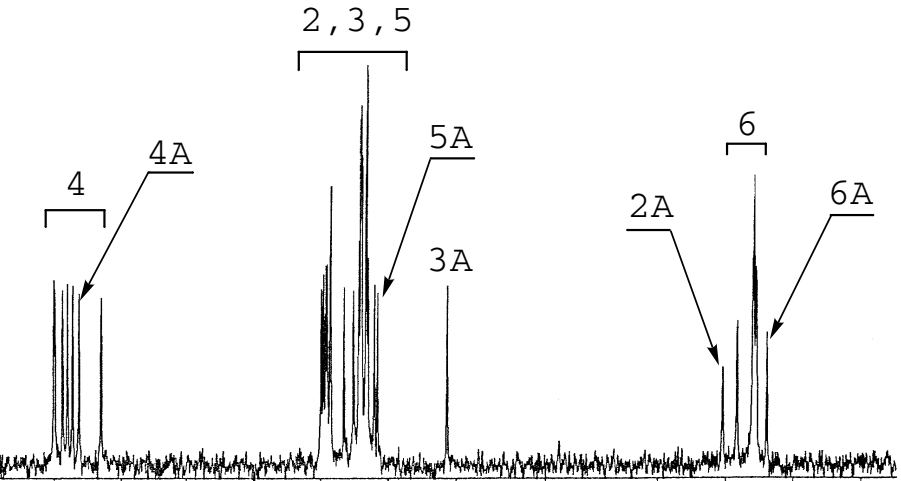

ppm

60

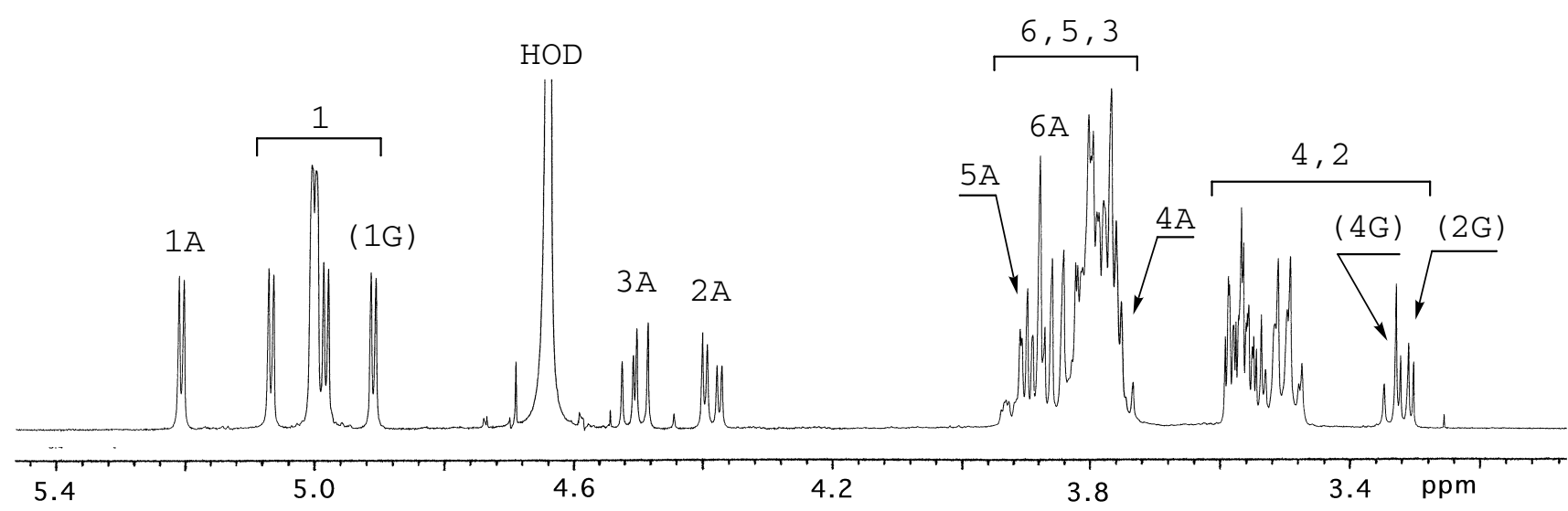

Fig. S3 ${ }^{1} \mathrm{H}(500 \mathrm{MHz})$ and ${ }^{13} \mathrm{C}$ NMR spectra $(125 \mathrm{MHz})$ of $2^{\mathrm{A}}$-deoxy- $2^{\mathrm{A}}$-imidazolyl- $\beta-\mathrm{CD} \mathbf{1 0}$ in $\mathrm{D}_{2} \mathrm{O}\left(\mathrm{CH}_{3} \mathrm{CN}\right.$ int $)$. 


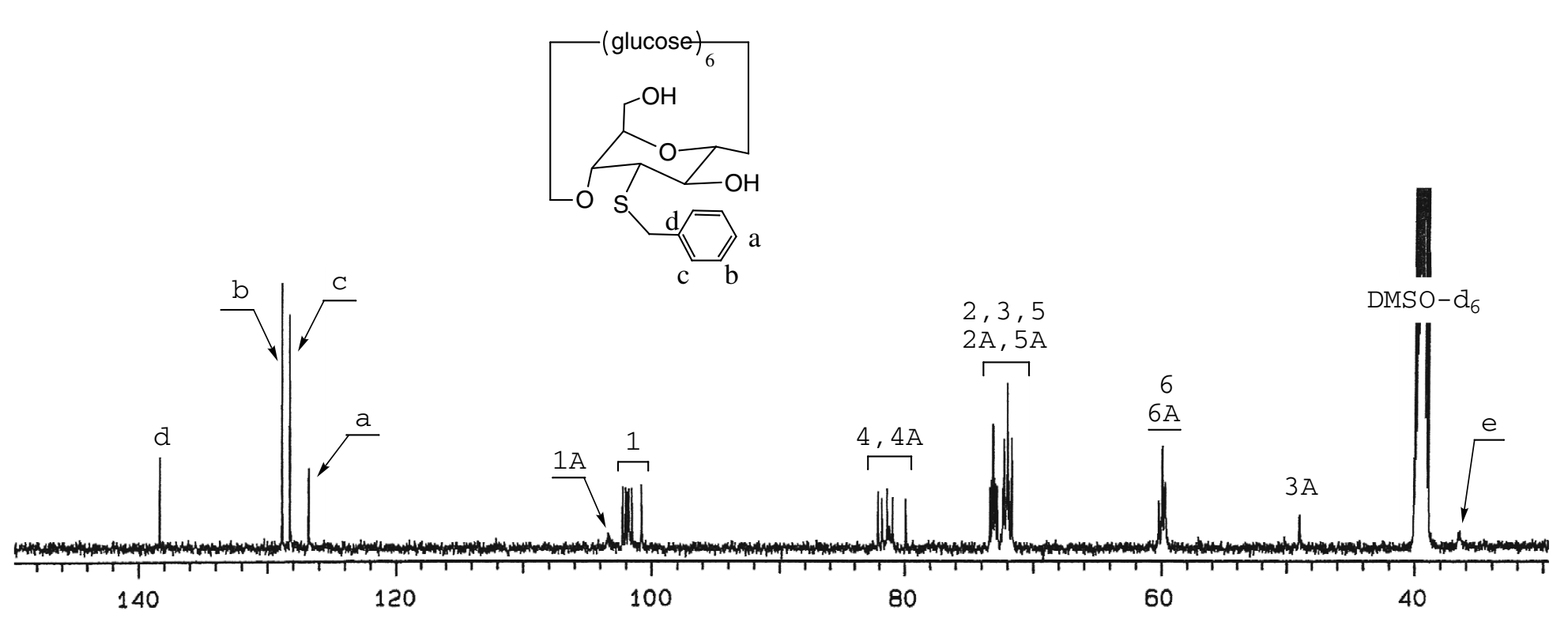

11

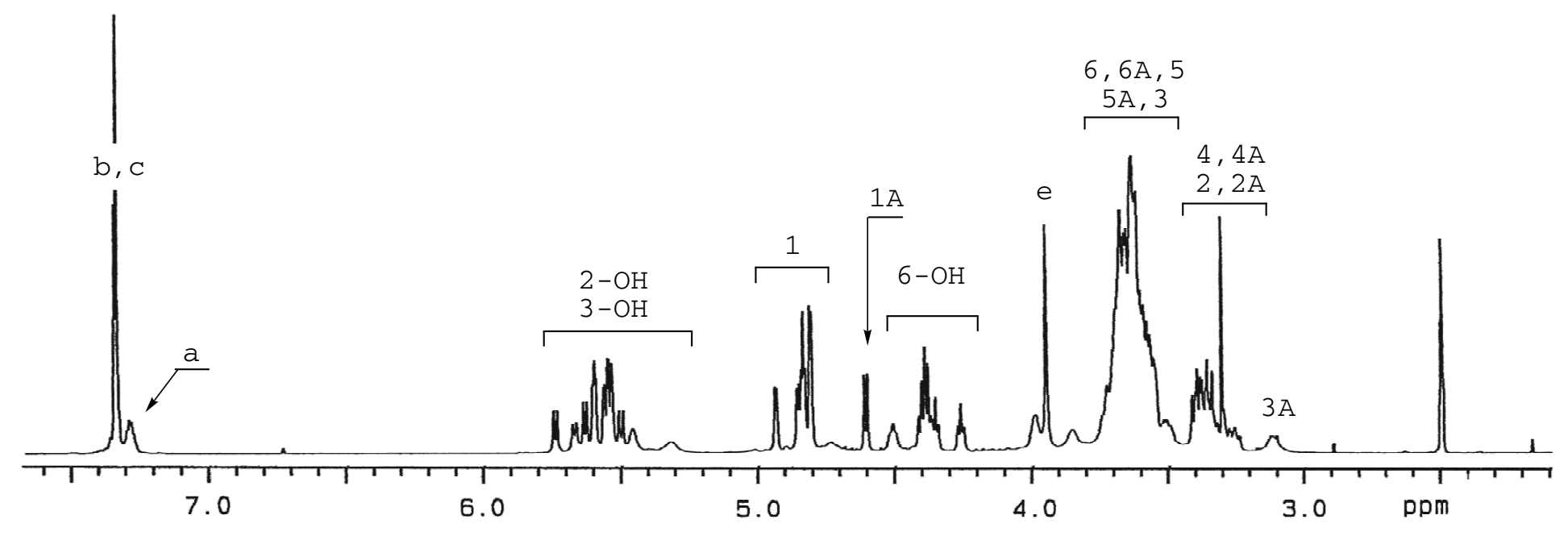

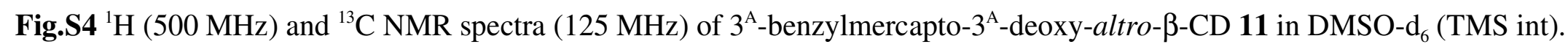



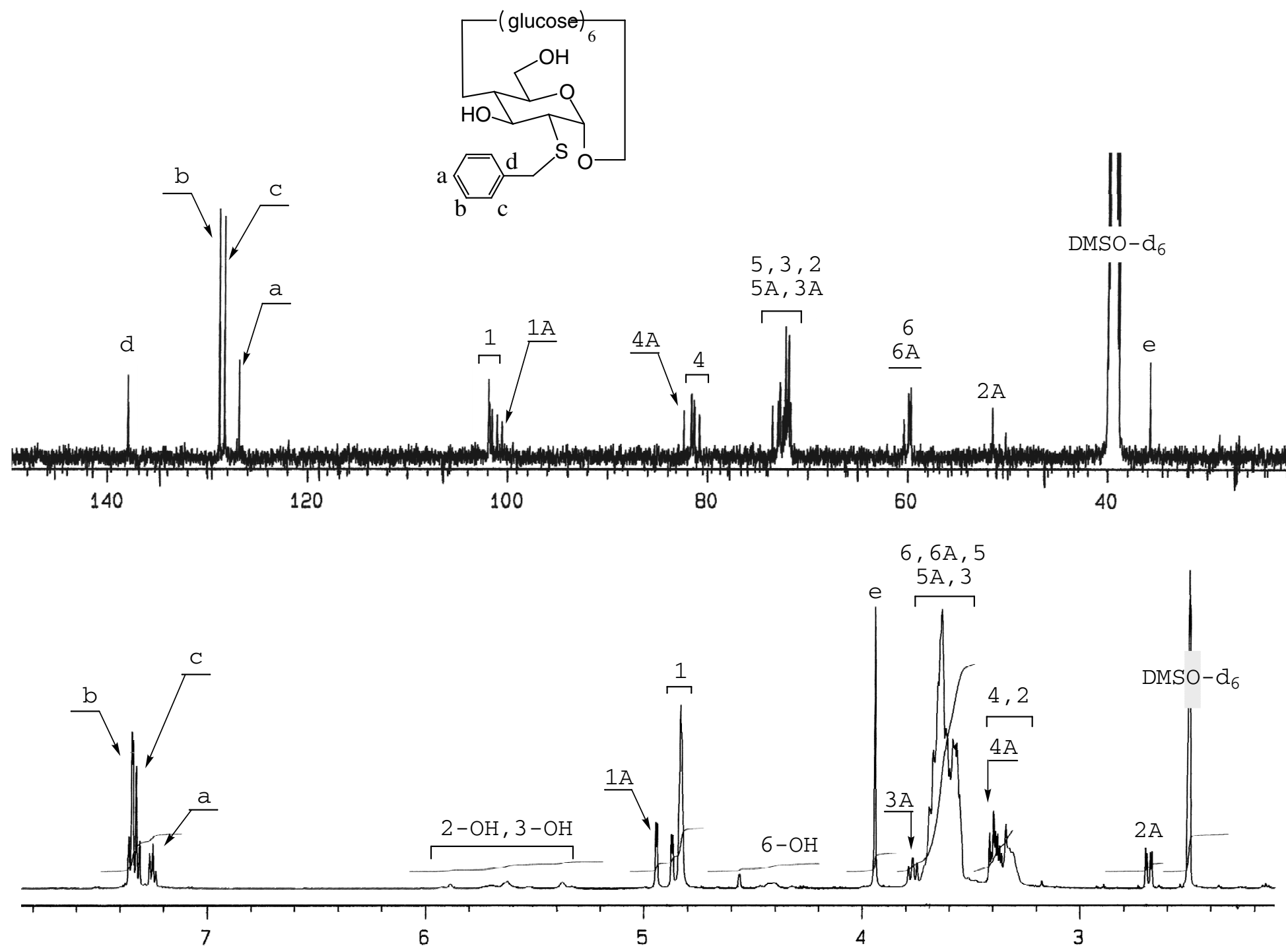

Fig. S5 ${ }^{1} \mathrm{H}(500 \mathrm{MHz})$ and ${ }^{13} \mathrm{C}$ NMR spectra $(125 \mathrm{MHz})$ of $2^{\mathrm{A}}$-benzylmercapto- $2^{\mathrm{A}}$-deoxy- $\beta$-CD 12 in DMSO-d 6 (TMS int, deuterated). 

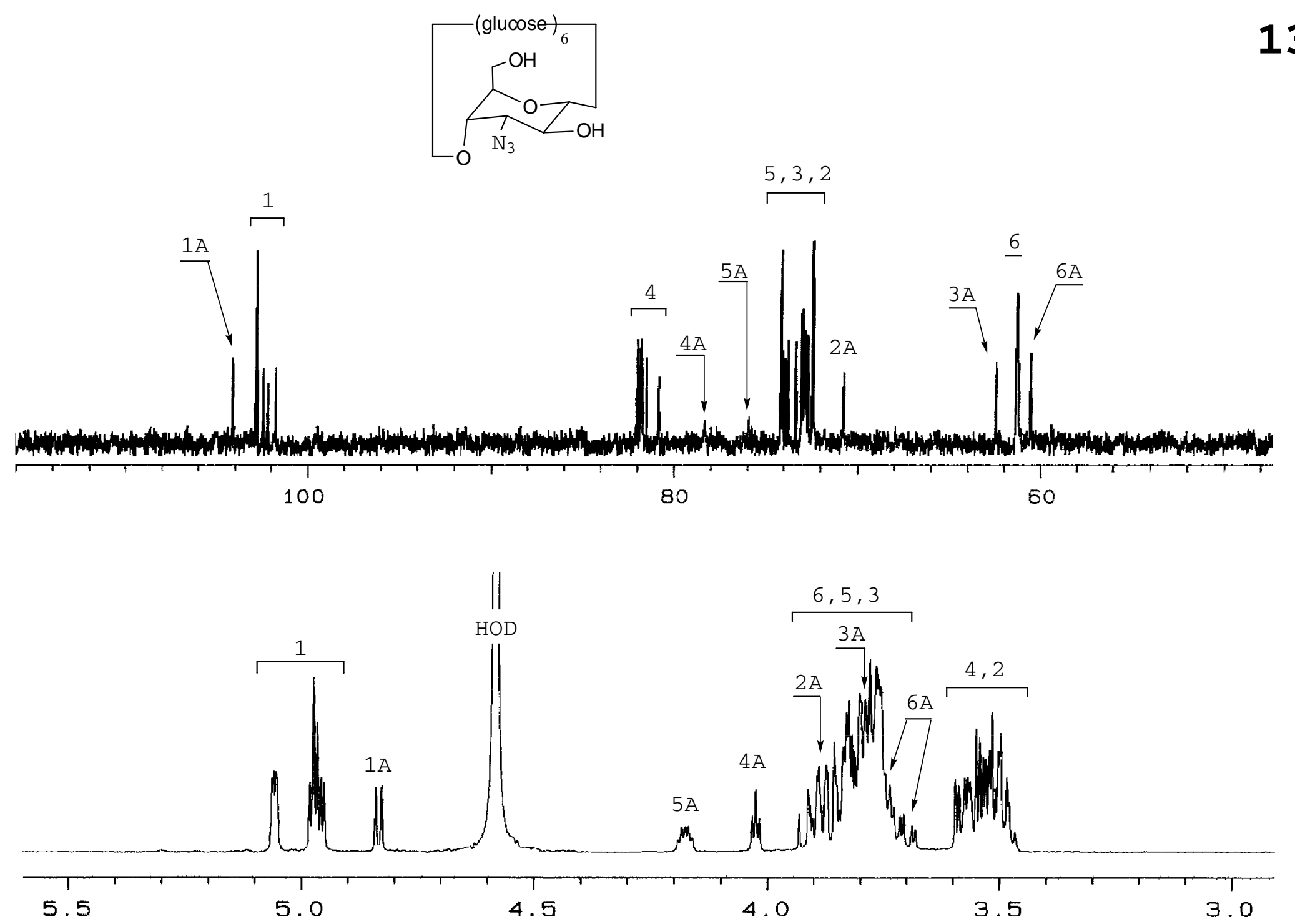

Fig. S6 ${ }^{1} \mathrm{H}(500 \mathrm{MHz})$ and ${ }^{13} \mathrm{C}$ NMR spectra $(125 \mathrm{MHz})$ of $3^{\mathrm{A}}$-azido- $3^{\mathrm{A}}$-deoxy-altro- $\beta$-CD 13 in $\mathrm{D}_{2} \mathrm{O}\left(\mathrm{CH}_{3} \mathrm{CN}\right.$ int $)$. 

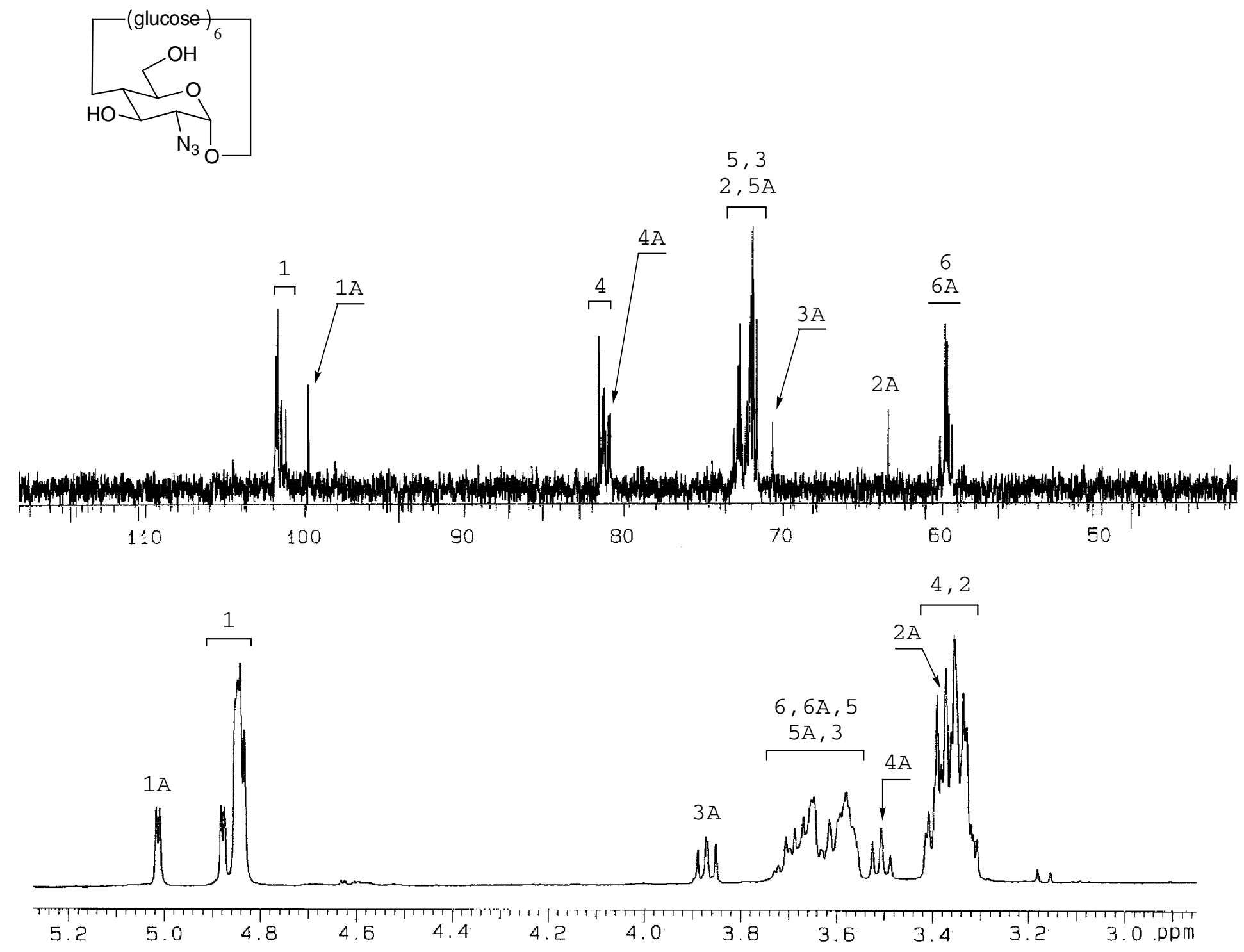

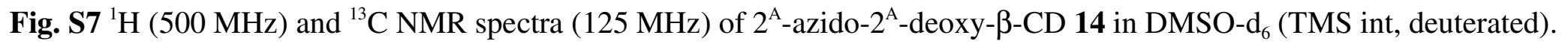



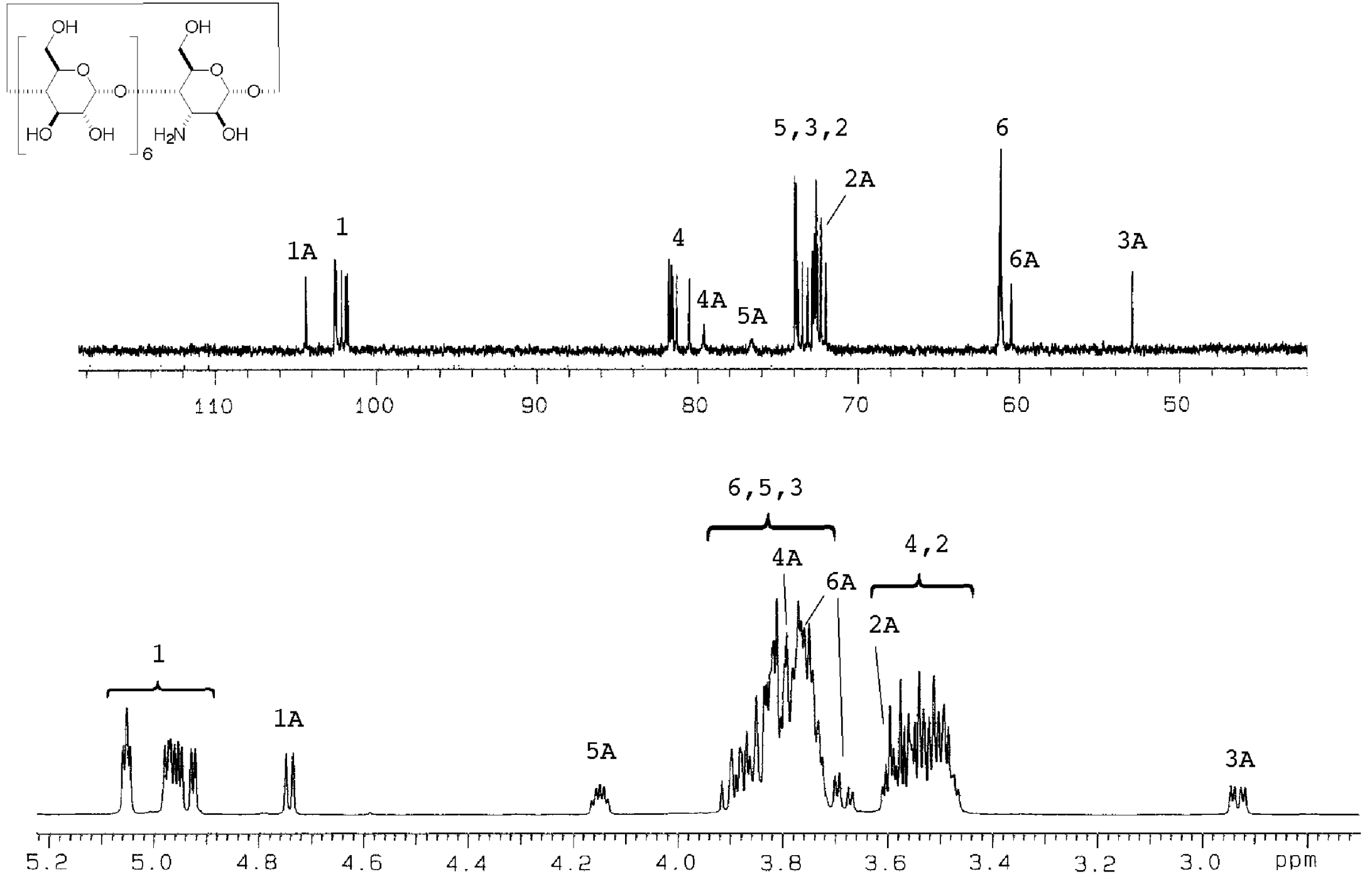

Fig. S8 ${ }^{1} \mathrm{H}(500 \mathrm{MHz})$ and ${ }^{13} \mathrm{C}$ NMR spectra $(125 \mathrm{MHz})$ of $3^{\mathrm{A}}$-amino- $3^{\mathrm{A}}$-deoxy-altro- $\beta$-CD 15 in $\mathrm{D}_{2} \mathrm{O}\left(\mathrm{CH}_{3} \mathrm{CN}\right.$ int $)$. 


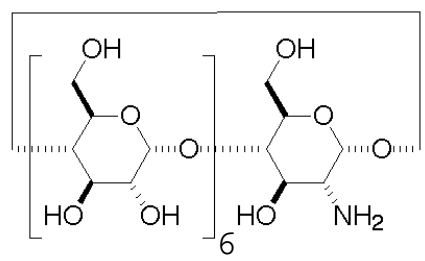

in DMSO-d 6 (int. TMS, $40^{\circ} \mathrm{C}$ )
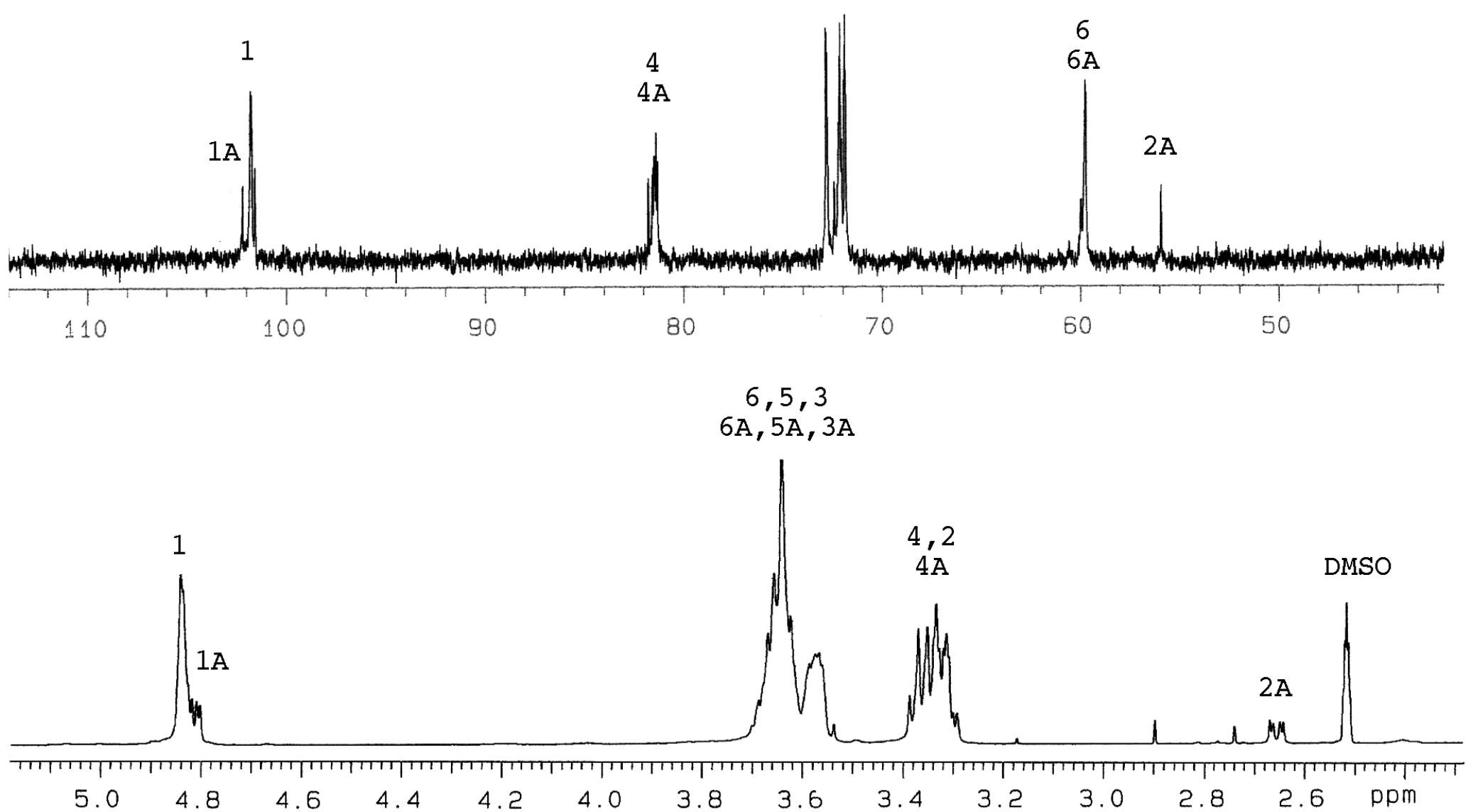

Fig. S9 ${ }^{1} \mathrm{H}(500 \mathrm{MHz})$ and ${ }^{13} \mathrm{C}$ NMR spectra $(125 \mathrm{MHz})$ of $2^{\mathrm{A}}$-amino- $2^{\mathrm{A}}$-deoxy- $\beta$-CD 16 in DMSO-d 6 (TMS int, deuterated). 

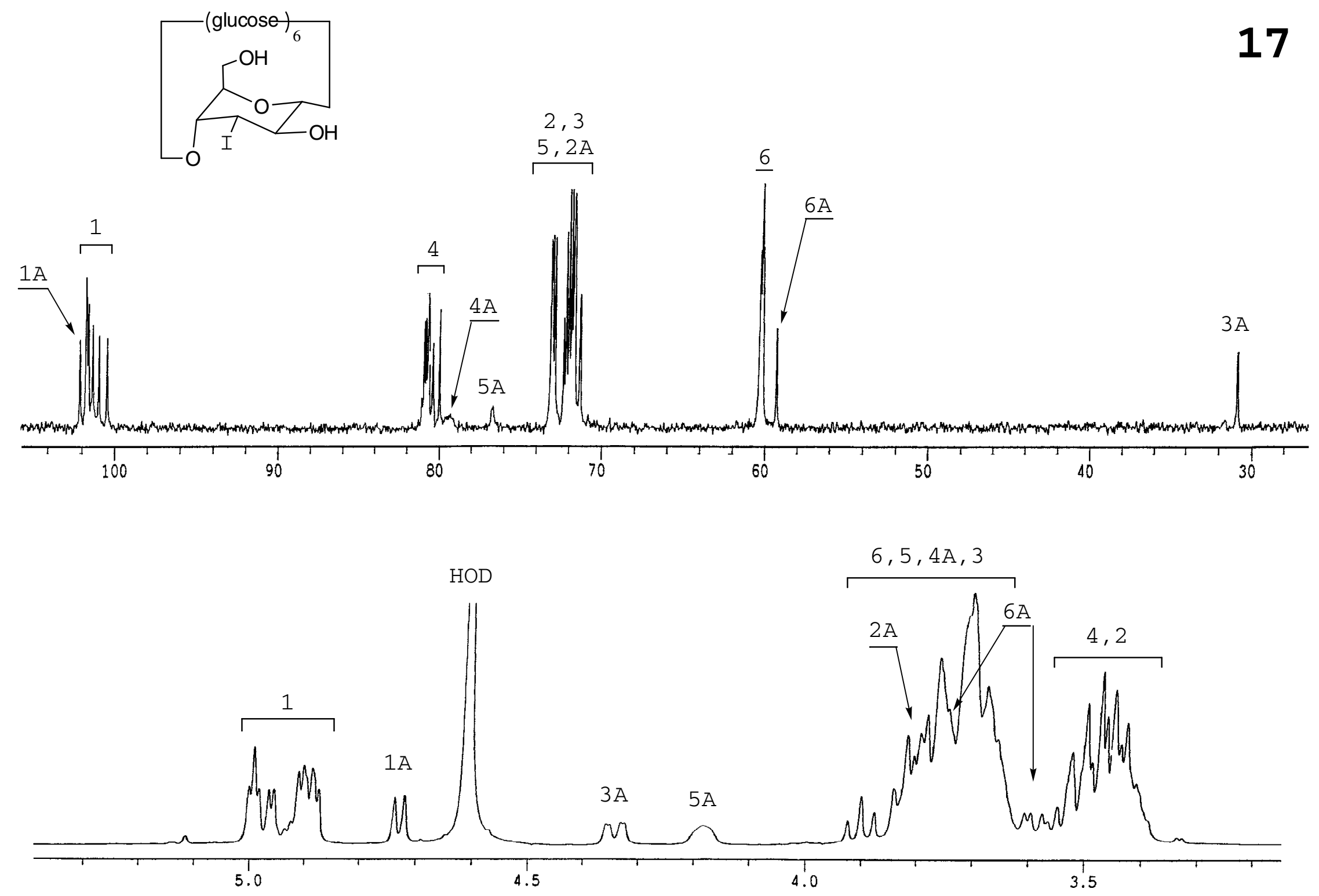

Fig. S10 ${ }^{1} \mathrm{H}(400 \mathrm{MHz})$ and ${ }^{13} \mathrm{C}$ NMR spectra $(100 \mathrm{MHz})$ of $3^{\mathrm{A}}$-deoxy- $3^{\mathrm{A}}$-iodo-altro- $\beta$-CD 17 in $\mathrm{D}_{2} \mathrm{O}\left(\mathrm{CH}_{3} \mathrm{CN}\right.$ int $)$. 

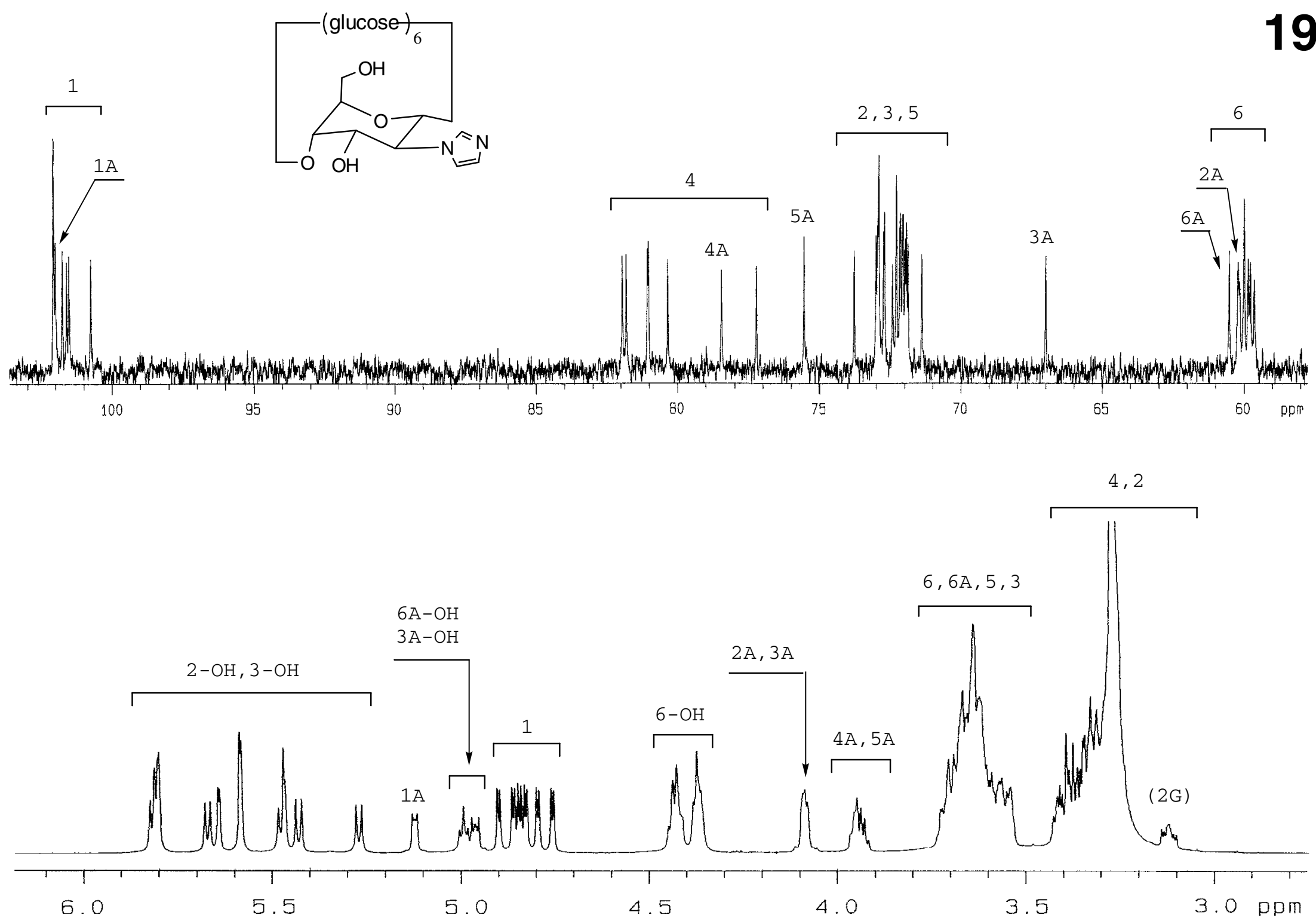

Fig. S11 ${ }^{1} \mathrm{H}(500 \mathrm{MHz})$ and ${ }^{13} \mathrm{C}$ NMR spectra $(125 \mathrm{MHz})$ of $2^{\mathrm{A}}$-deoxy-2 $2^{\mathrm{A}}$-imidazolyl-altro- $\beta-\mathrm{CD} 19$ in DMSO-d ${ }_{6}$ (TMS int). 


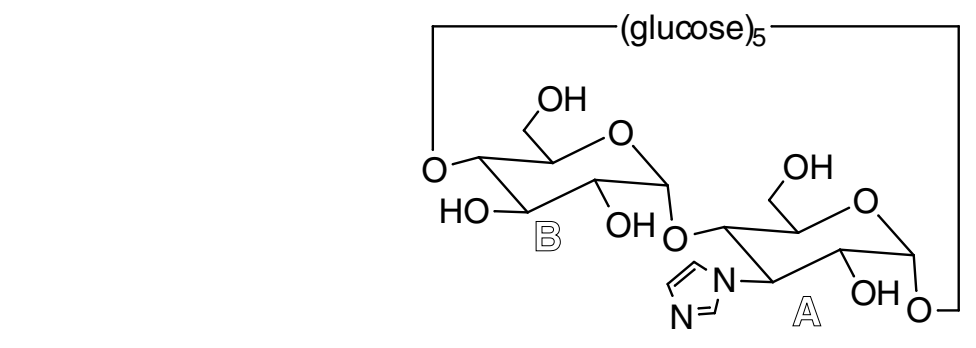

20
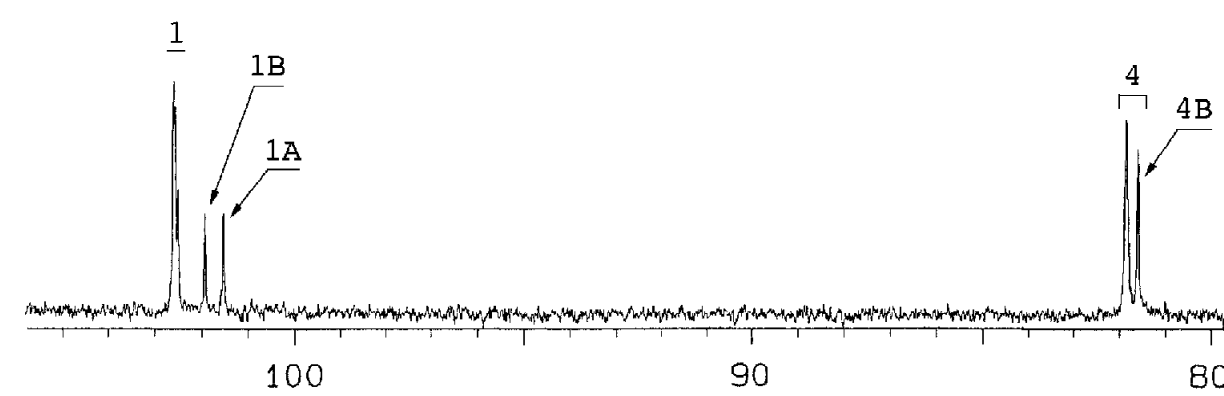

80

60

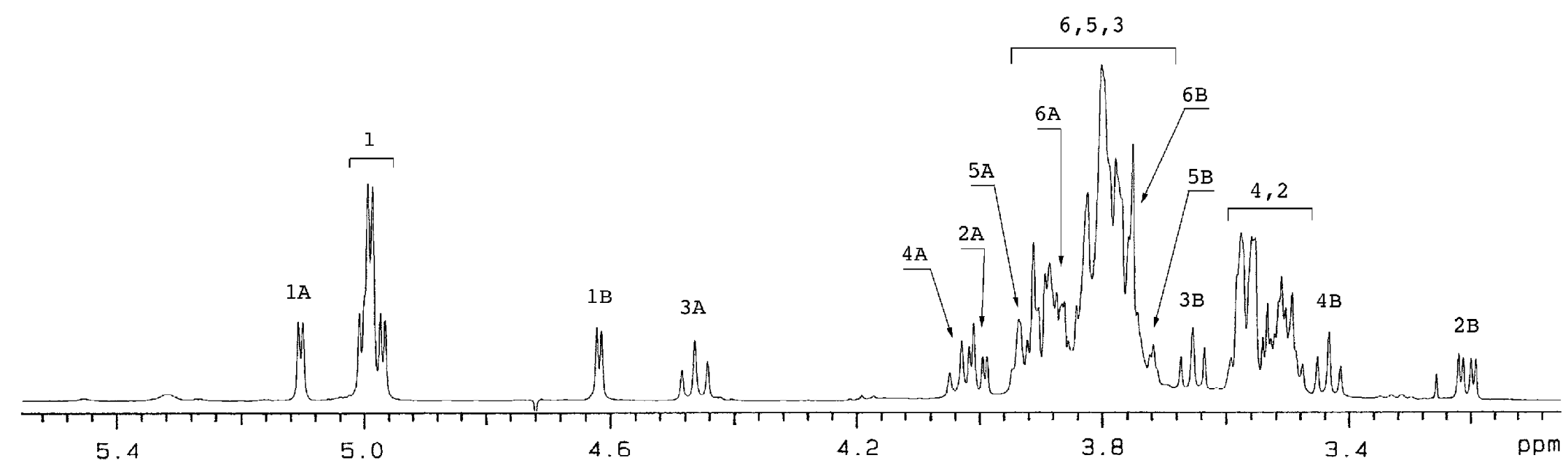

Fig. S12 ${ }^{1} \mathrm{H}(500 \mathrm{MHz})$ and ${ }^{13} \mathrm{C}$ NMR spectra $(125 \mathrm{MHz})$ of $3^{\mathrm{A}}$-deoxy- $3^{\mathrm{A}}$-imidazolyl- $\beta-\mathrm{CD} 20$ in $\mathrm{D}_{2} \mathrm{O}\left(\mathrm{CH}_{3} \mathrm{CN}\right.$ int $)$. 

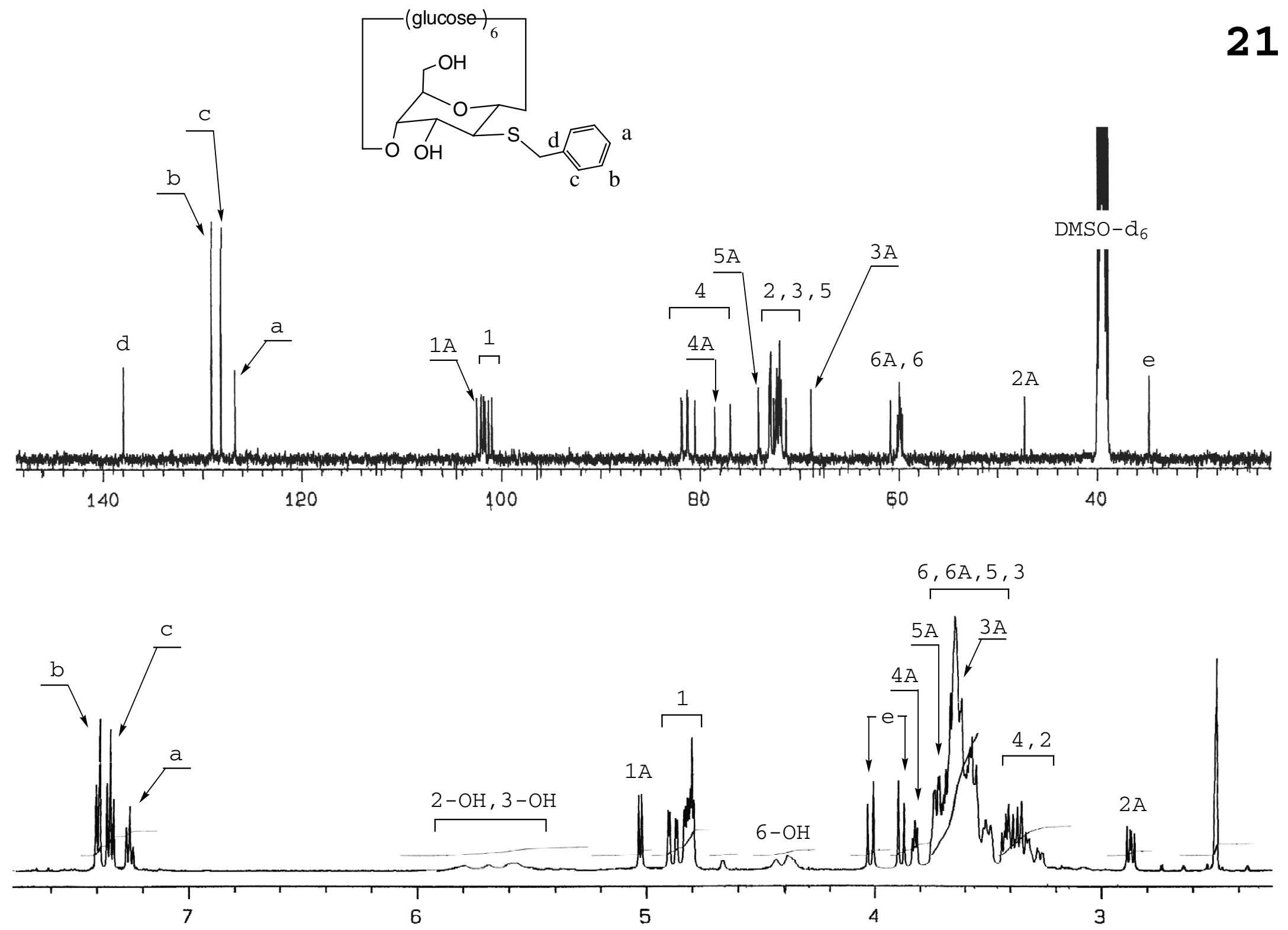

Fig. $\mathbf{S 1 3}{ }^{1} \mathrm{H}(500 \mathrm{MHz})$ and ${ }^{13} \mathrm{C}$ NMR spectra $(125 \mathrm{MHz})$ of $2^{\mathrm{A}}$-benzylmercapto- $2^{\mathrm{A}}$-deoxy-altro- $\beta$-CD 21 in DMSO-d ${ }_{6}$ (TMS int, deuterated). 

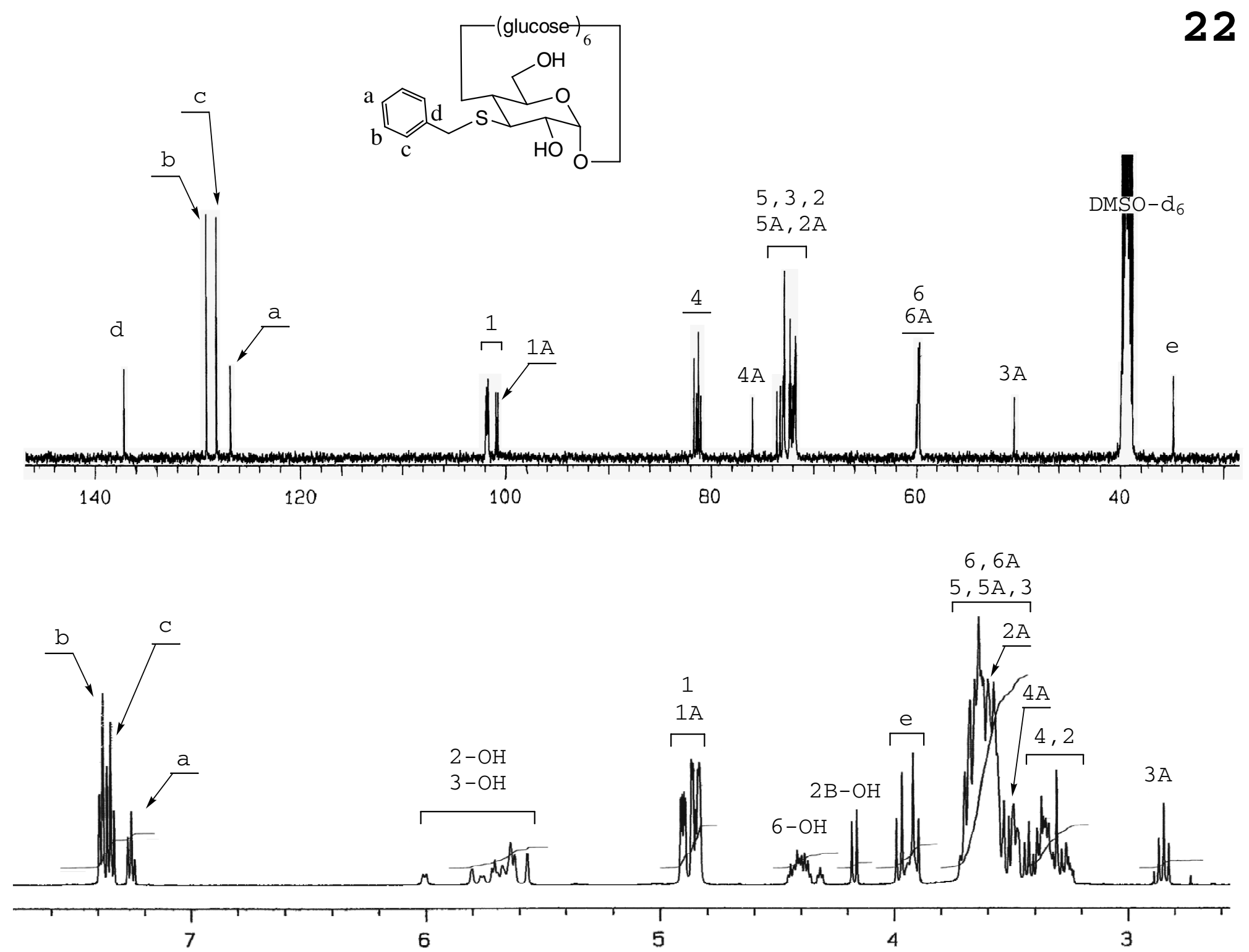

Fig. $\mathbf{S 1 4}{ }^{1} \mathrm{H}(500 \mathrm{MHz})$ and ${ }^{13} \mathrm{C}$ NMR spectra $(125 \mathrm{MHz})$ of $3^{\mathrm{A}}$-benzylmercapto- $3^{\mathrm{A}}$-deoxy- $\beta$-CD 22 in DMSO-d ${ }_{6}$ (TMS int, deuterated) 


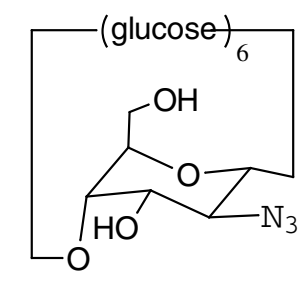

23

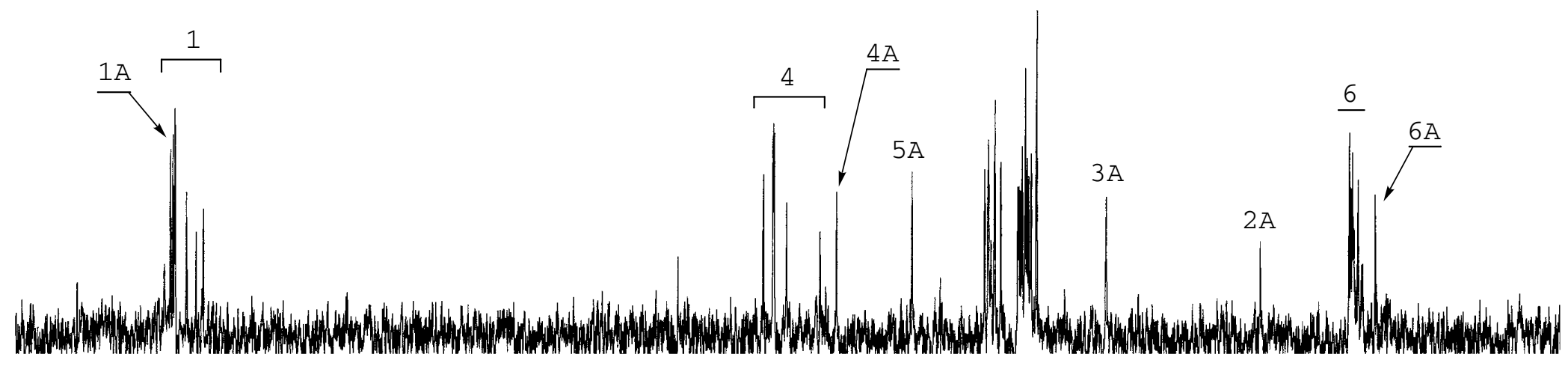

100

90

80

70

60

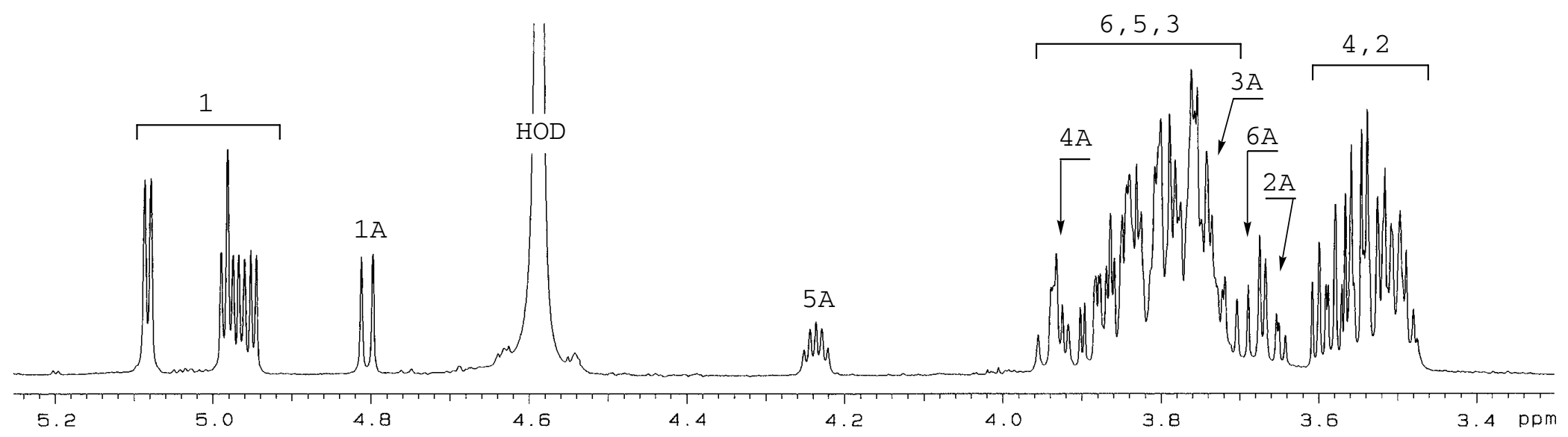

Fig. $\mathbf{S 1 5}{ }^{1} \mathrm{H}(500 \mathrm{MHz})$ and ${ }^{13} \mathrm{C}$ NMR spectra $(125 \mathrm{MHz})$ of $2^{\mathrm{A}}$-azido- $2^{\mathrm{A}}$-deoxy-altro- $\beta$-CD 23 in $\mathrm{D}_{2} \mathrm{O}\left(\mathrm{CH}_{3} \mathrm{CN}\right.$ int $)$. 

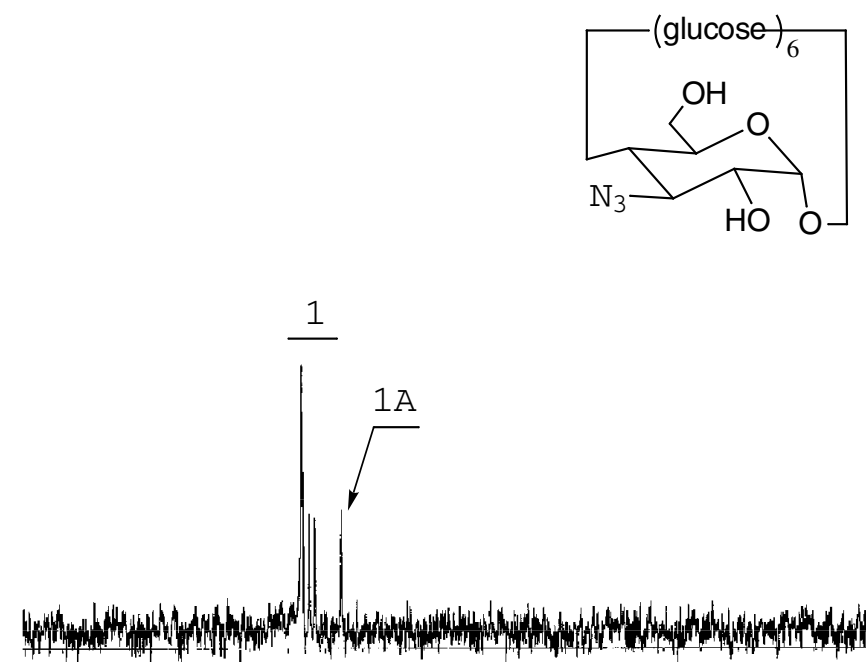

100

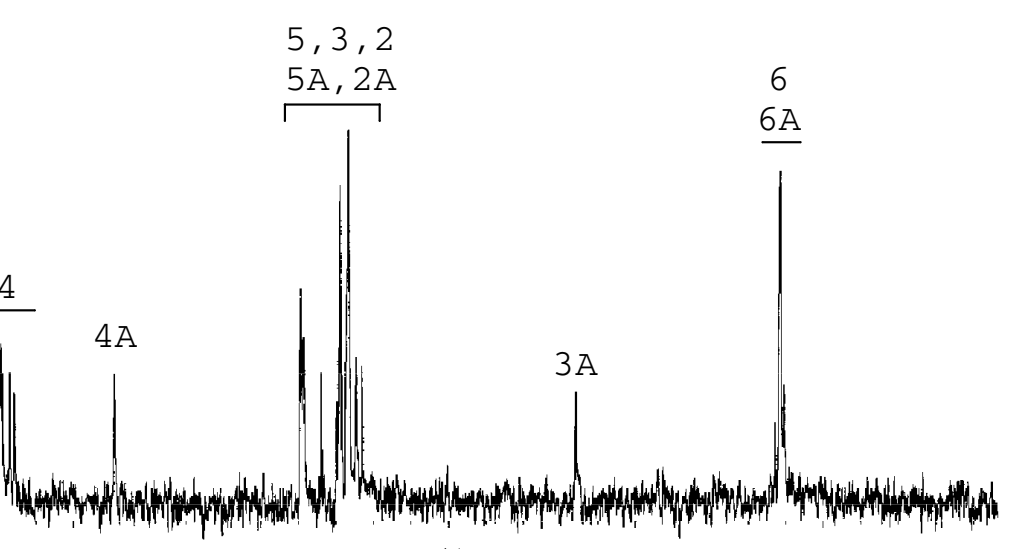

70

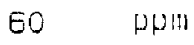

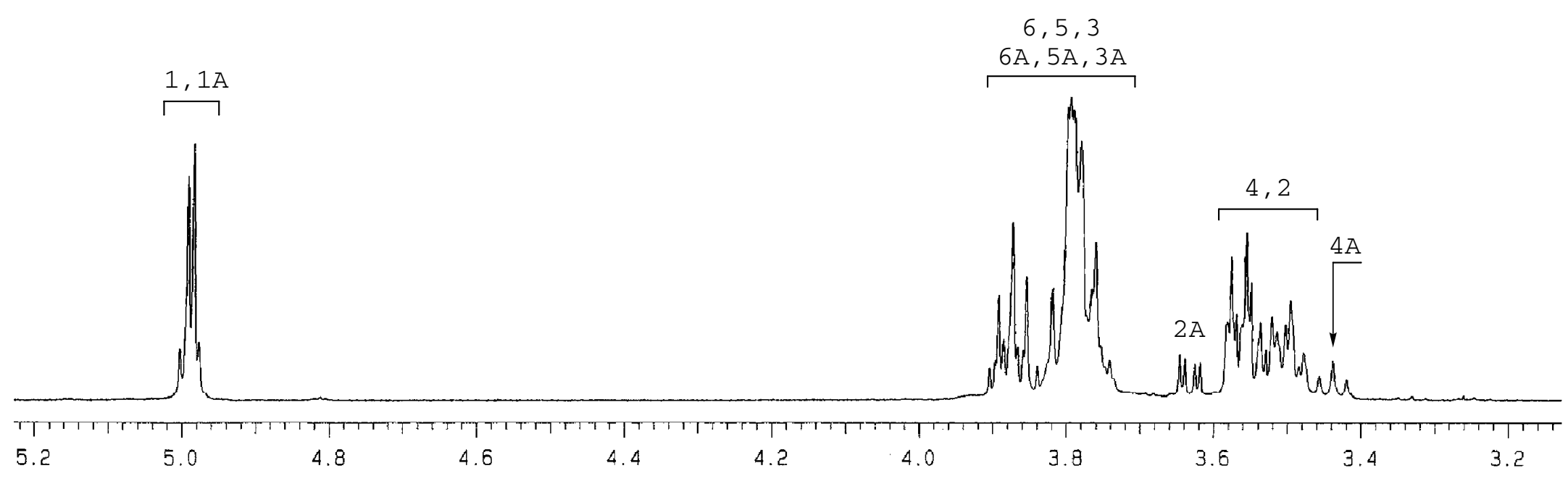

Fig. $\mathbf{S 1 6}{ }^{1} \mathrm{H}(500 \mathrm{MHz})$ and ${ }^{13} \mathrm{C}$ NMR spectra $(125 \mathrm{MHz})$ of $3^{\mathrm{A}}$-azido- $3^{\mathrm{A}}$-deoxy- $\beta-\mathrm{CD} 24$ in $\mathrm{D}_{2} \mathrm{O}\left(\mathrm{CH}_{3} \mathrm{CN}\right.$ int $)$. 

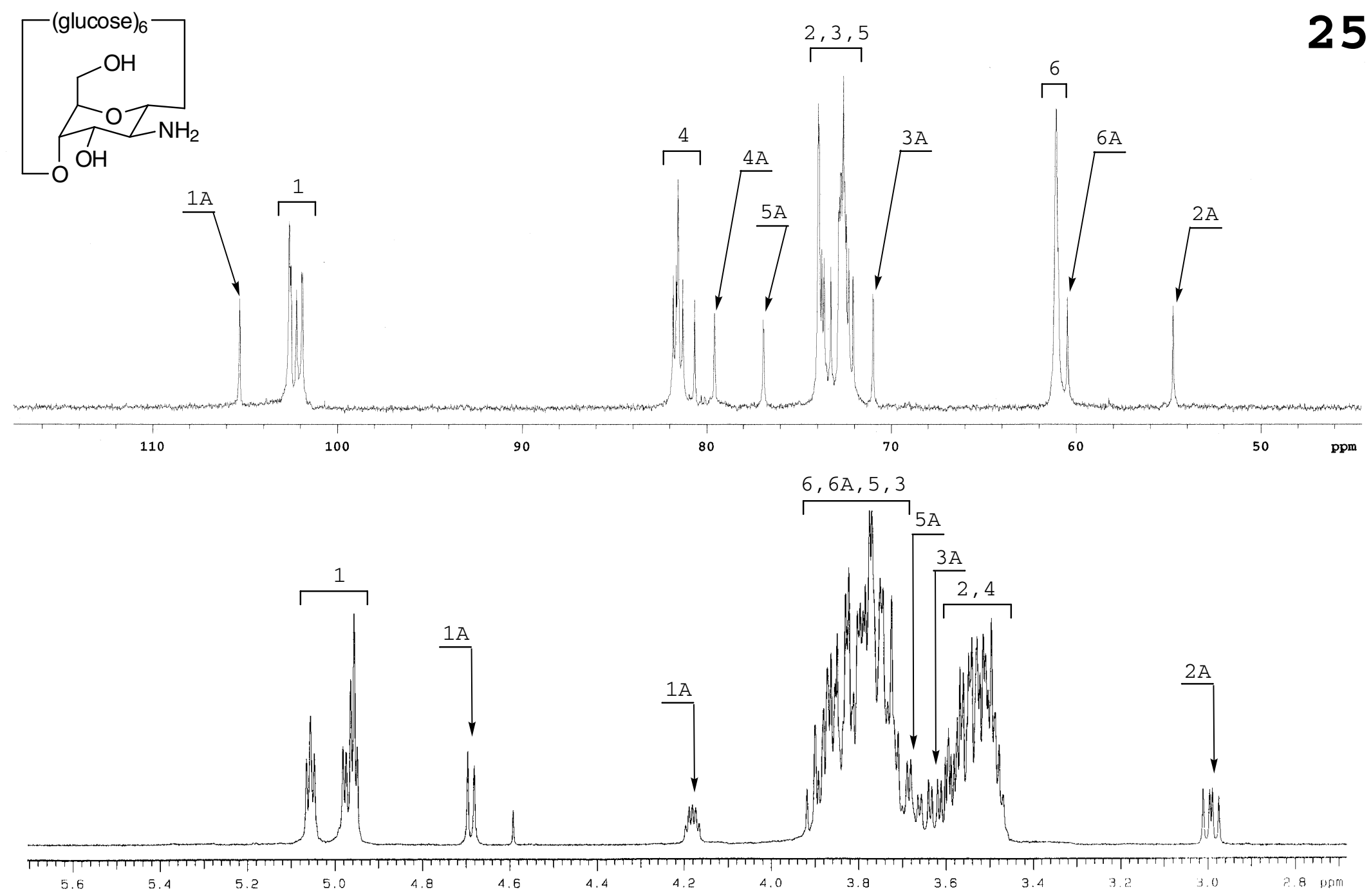

Fig. $\mathbf{S 1 7}{ }^{1} \mathrm{H}(500 \mathrm{MHz})$ and ${ }^{13} \mathrm{C}$ NMR spectra $(125 \mathrm{MHz})$ of $2^{\mathrm{A}}$-amino- $2^{\mathrm{A}}$-deoxy-altro- $\beta$-CD 25 in $\mathrm{D}_{2} \mathrm{O}\left(\mathrm{CH}_{3} \mathrm{CN}\right.$ int $)$. 


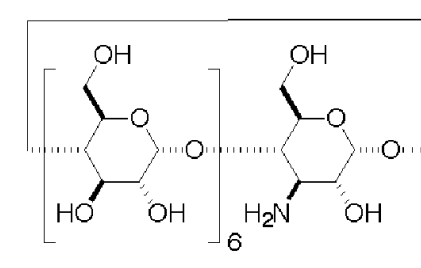

in DMSO-d 6 (int. TMS, $40^{\circ} \mathrm{C}$ )
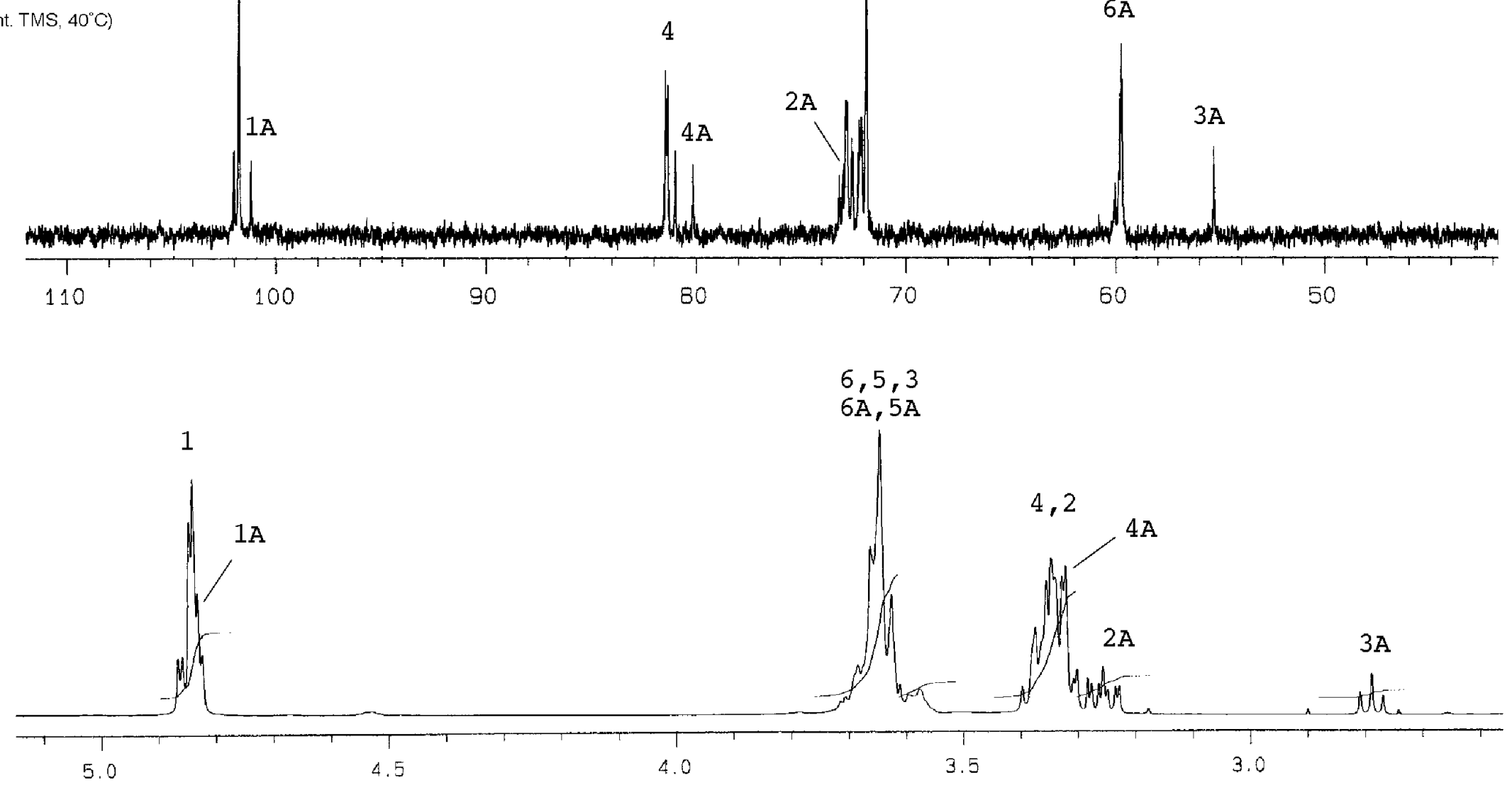

Fig. S18 ${ }^{1} \mathrm{H}(500 \mathrm{MHz})$ and ${ }^{13} \mathrm{C}$ NMR spectra $(125 \mathrm{MHz})$ of $3^{\mathrm{A}}$-amino- $3^{\mathrm{A}}$-deoxy- $\beta$-CD 26 in DMSO-d 6 (TMS int, deuterated). 

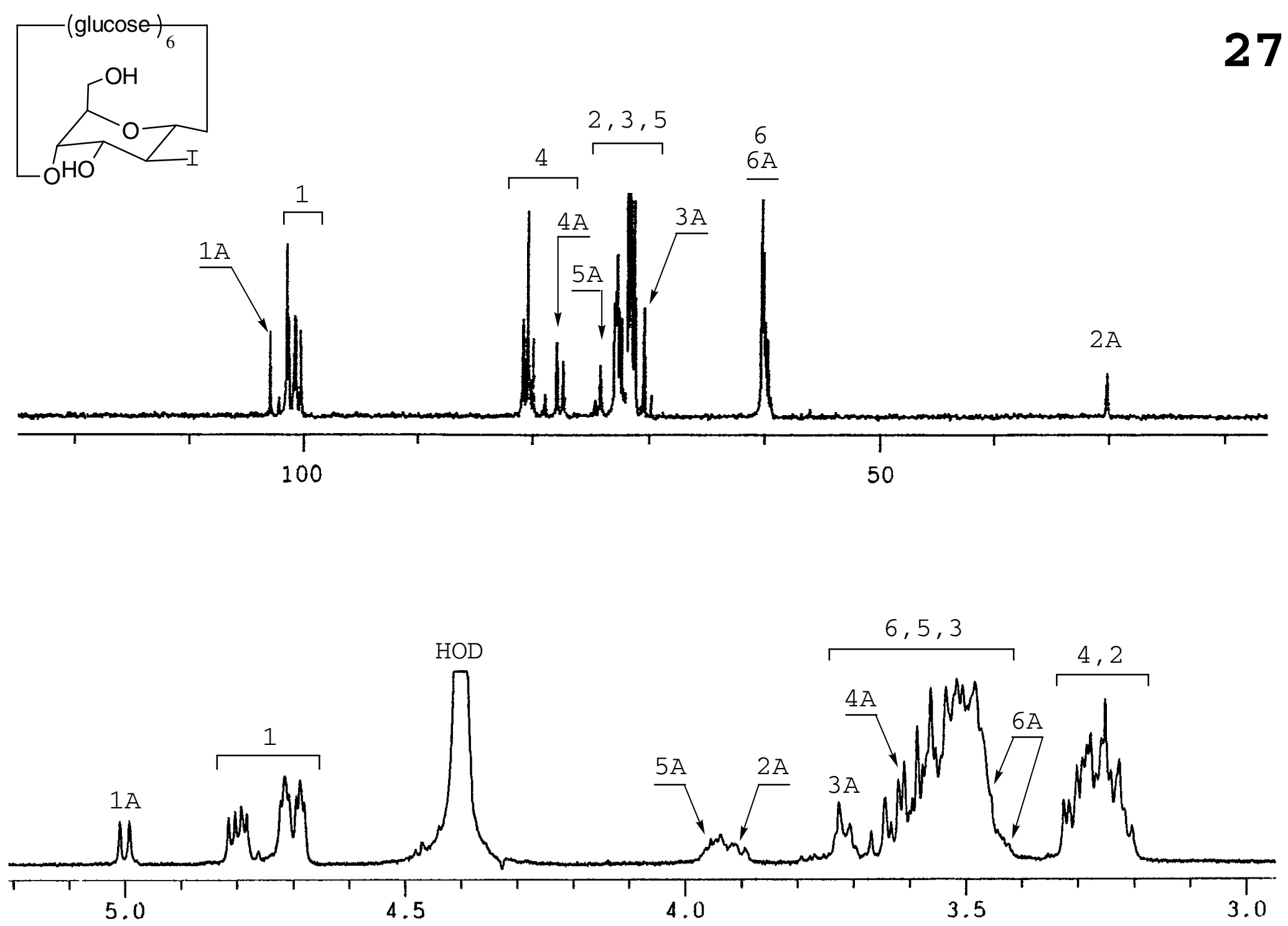

Fig. S19 ${ }^{1} \mathrm{H}(400 \mathrm{MHz})$ and ${ }^{13} \mathrm{C}$ NMR spectra $(100 \mathrm{MHz})$ of $2^{\mathrm{A}}$-deoxy- $2^{\mathrm{A}}$-iodo-altro- $\beta$-CD 27 in $\mathrm{D}_{2} \mathrm{O}\left(\mathrm{CH}_{3} \mathrm{CN}\right.$ int $)$. 

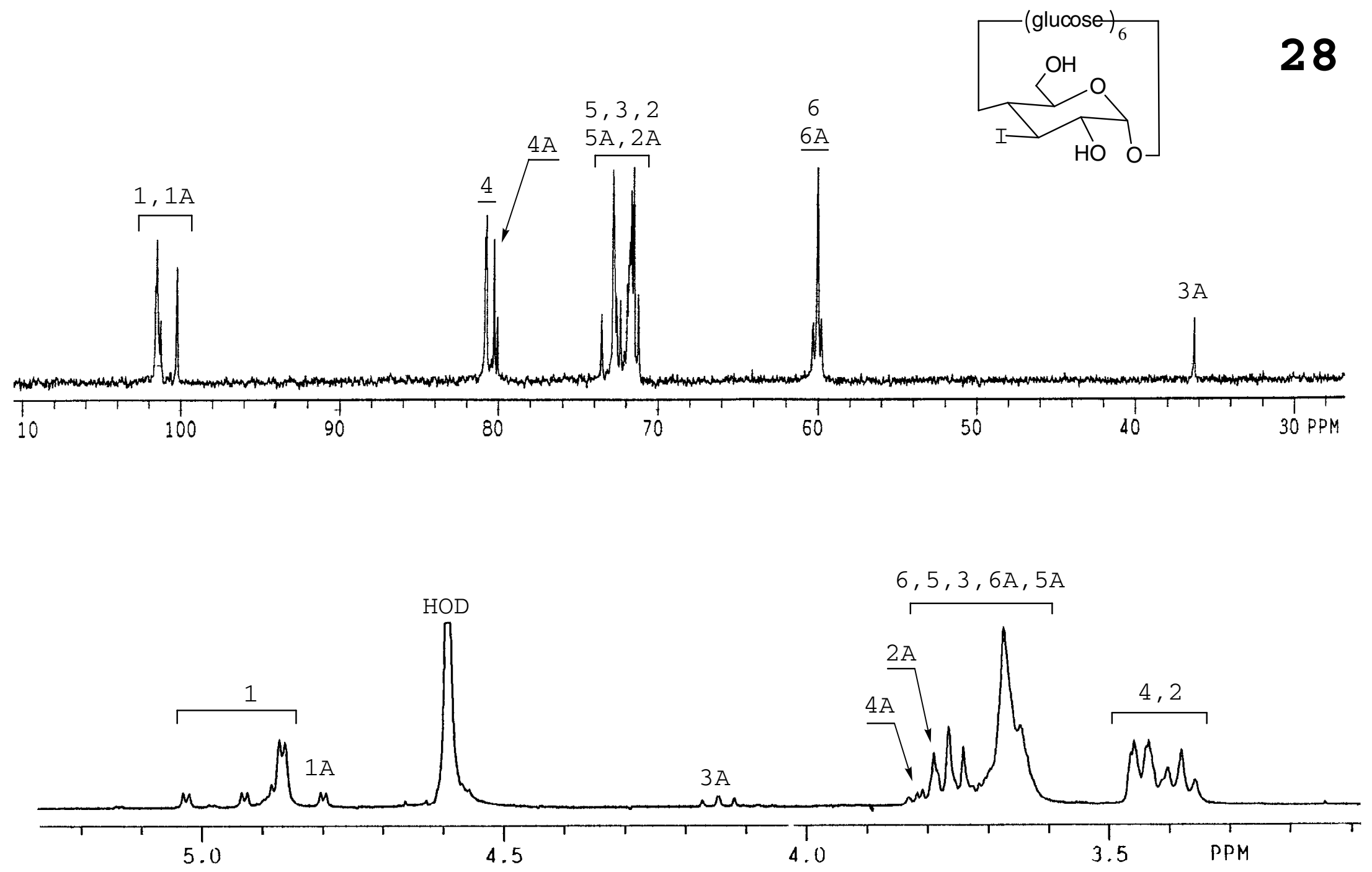

Fig. S20 ${ }^{1} \mathrm{H}(400 \mathrm{MHz})$ and ${ }^{13} \mathrm{C}$ NMR spectra $(100 \mathrm{MHz})$ of $3^{\mathrm{A}}$-deoxy- $3^{\mathrm{A}}$-iodo- $\beta$-CD 28 in $\mathrm{D}_{2} \mathrm{O}\left(\mathrm{CH}_{3} \mathrm{CN}\right.$ int $)$. 


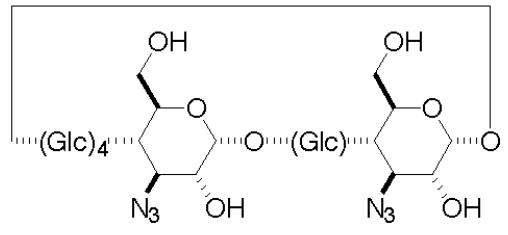

in $\mathrm{DMSO}^{-\mathrm{d}_{6}}$ (int. TMS, $40^{\circ} \mathrm{C}$ )

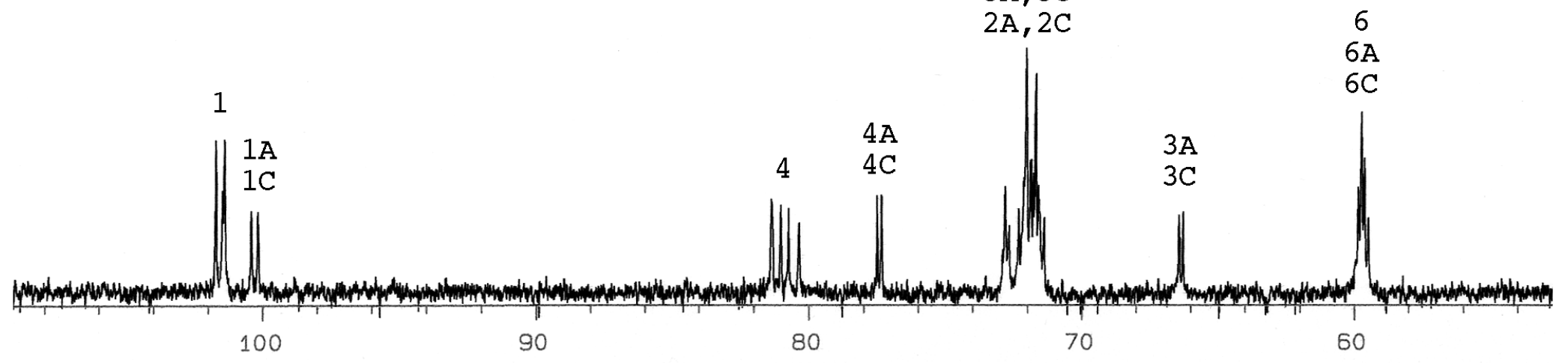

80

60
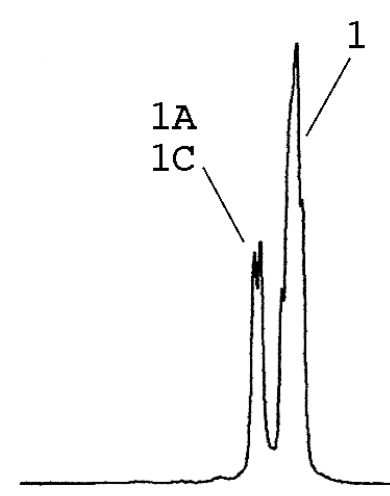

5.0

4. 8

4.6

4. 4

4.0

3.8

3.6

\section{$6.5,3$}

4,2
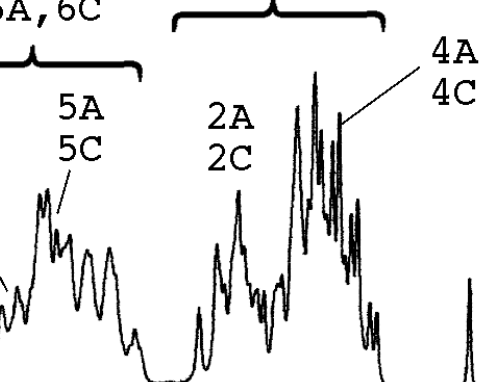

Fig. S21 ${ }^{1} \mathrm{H}(500 \mathrm{MHz})$ and ${ }^{13} \mathrm{C}$ NMR spectra $(125 \mathrm{MHz})$ of $3^{\mathrm{A}}, 3^{\mathrm{C}}$-diazido- $3^{\mathrm{A}}, 3^{\mathrm{C}}$-dideoxy- $\beta$-CD 32 in DMSO- $\mathrm{d}_{6}$ (TMS int, deuterated). 


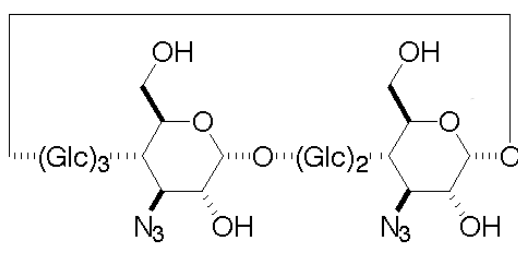

in DMSO-d $d_{6}$ (int. TMS, $40^{\circ} \mathrm{C}$ )

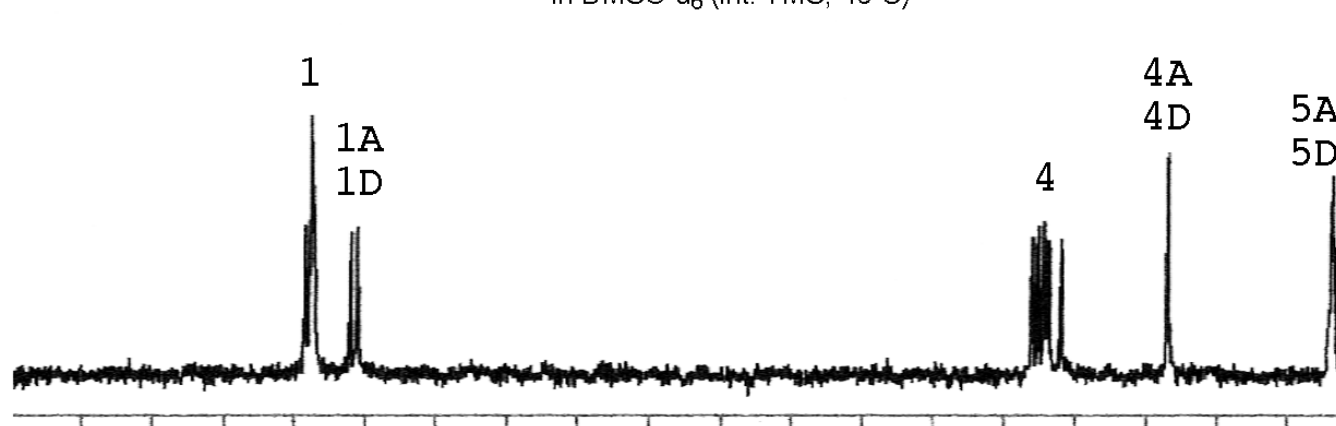

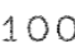
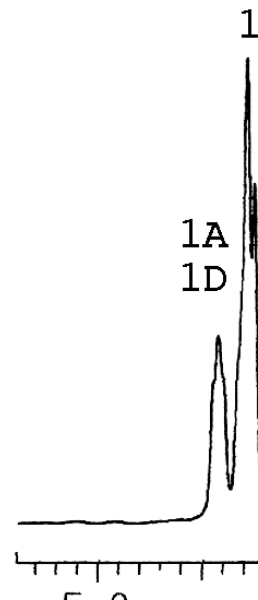

100

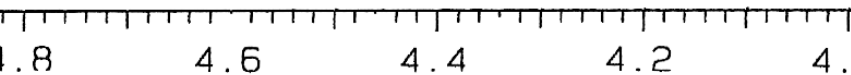

80
$5,3,2$

$2 \mathrm{~A}, 2 \mathrm{D}$

6

6

$6 \mathrm{D}$

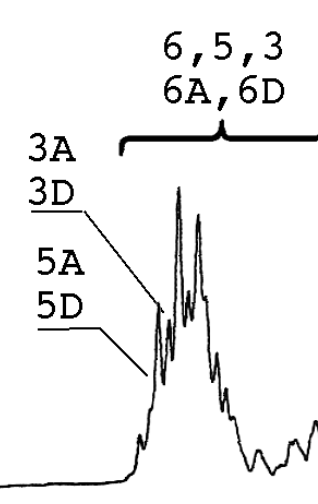

$, 5,3$
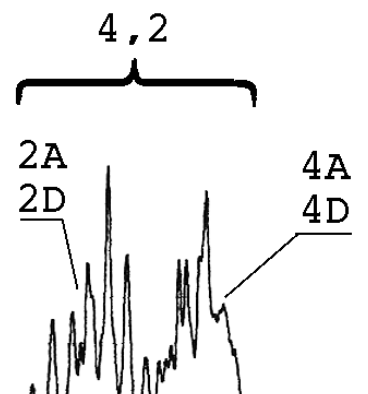

Fig. S22 ${ }^{1} \mathrm{H}(500 \mathrm{MHz})$ and ${ }^{13} \mathrm{C}$ NMR spectra $(125 \mathrm{MHz})$ of $3^{\mathrm{A}}, 3^{\mathrm{D}}$-diazido- $3^{\mathrm{A}}, 3^{\mathrm{D}}$-dideoxy- $\beta$-CD 33 in DMSO-d 6 (TMS int, deuterated). 

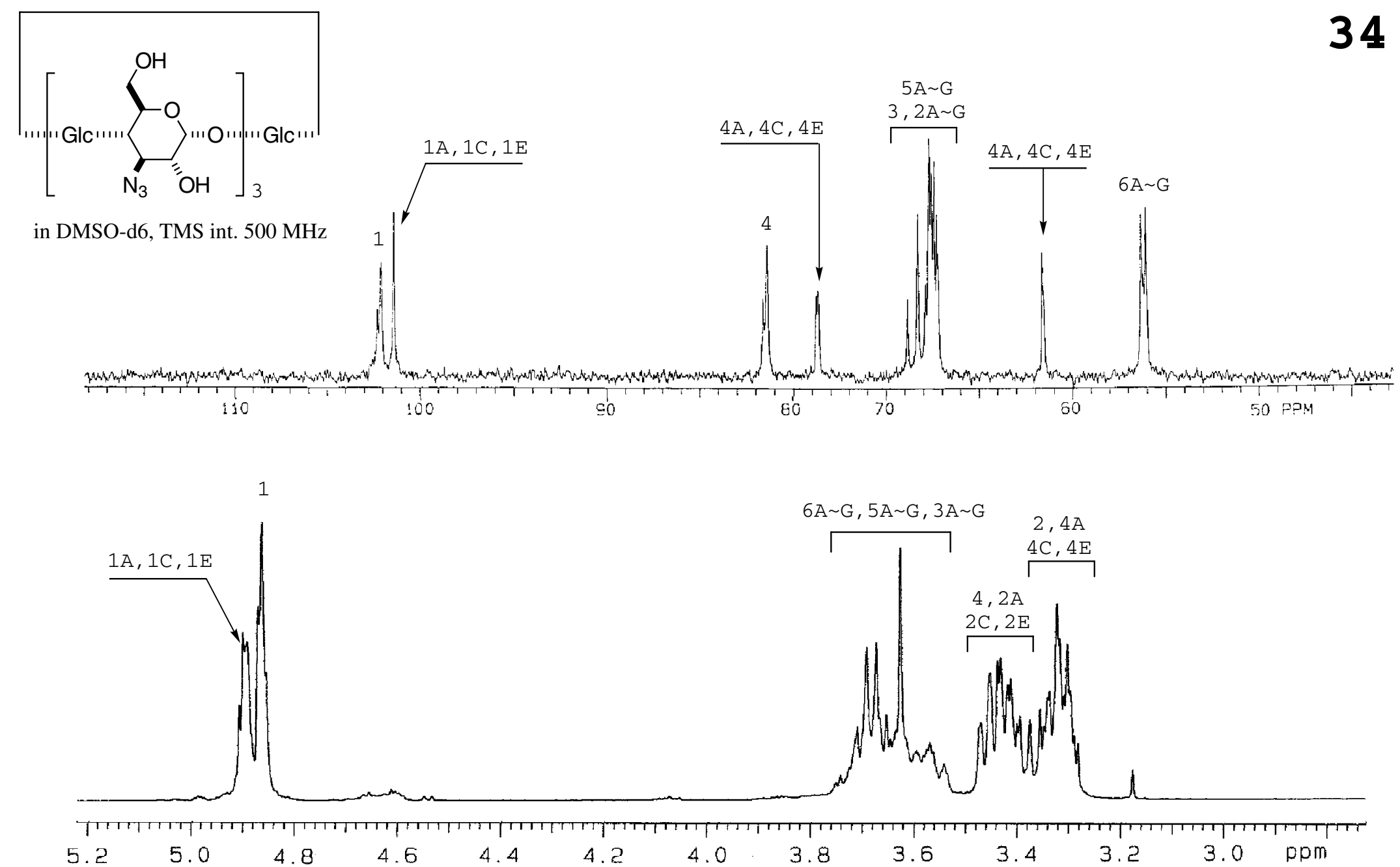

Fig. S23 ${ }^{1} \mathrm{H}(500 \mathrm{MHz})$ and ${ }^{13} \mathrm{C}$ NMR spectra $(125 \mathrm{MHz})$ of $3^{\mathrm{A}}, 3^{\mathrm{C}}, 3^{\mathrm{E}}$-triazido- $3^{\mathrm{A}}, 3^{\mathrm{C}}, 3^{\mathrm{E}}$-trideoxy- $\beta-\mathrm{CD} 34$ in DMSO-d 6 (TMS int, deuterated). 

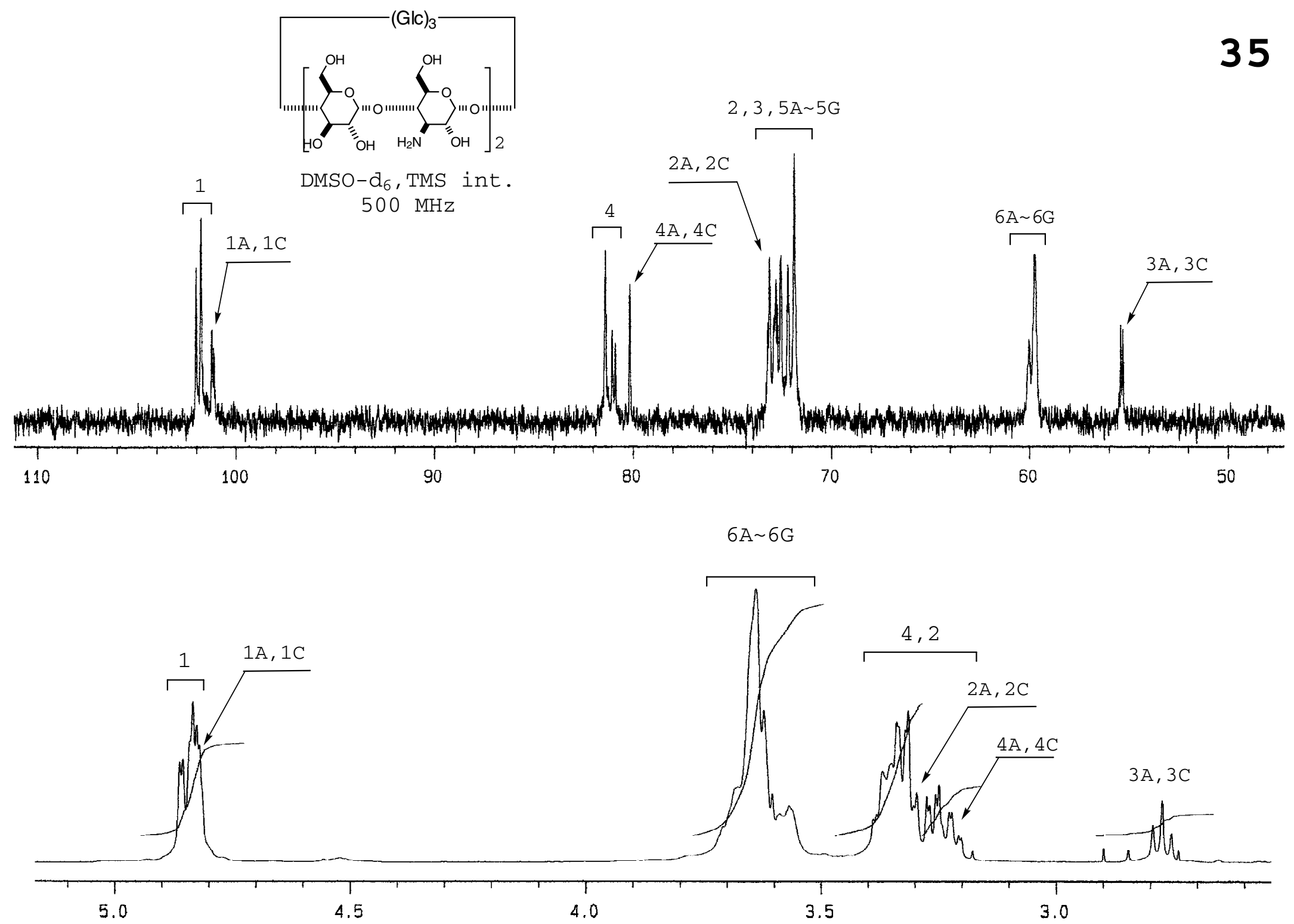

Fig. S24 ${ }^{1} \mathrm{H}(500 \mathrm{MHz})$ and ${ }^{13} \mathrm{C}$ NMR spectra $(125 \mathrm{MHz})$ of $3^{\mathrm{A}}, 3^{\mathrm{C}}$-diamino- $3^{\mathrm{A}}, 3^{\mathrm{C}}$-dideoxy- $\beta$-CD 35 in DMSO-d 6 (TMS int, deuterated). 

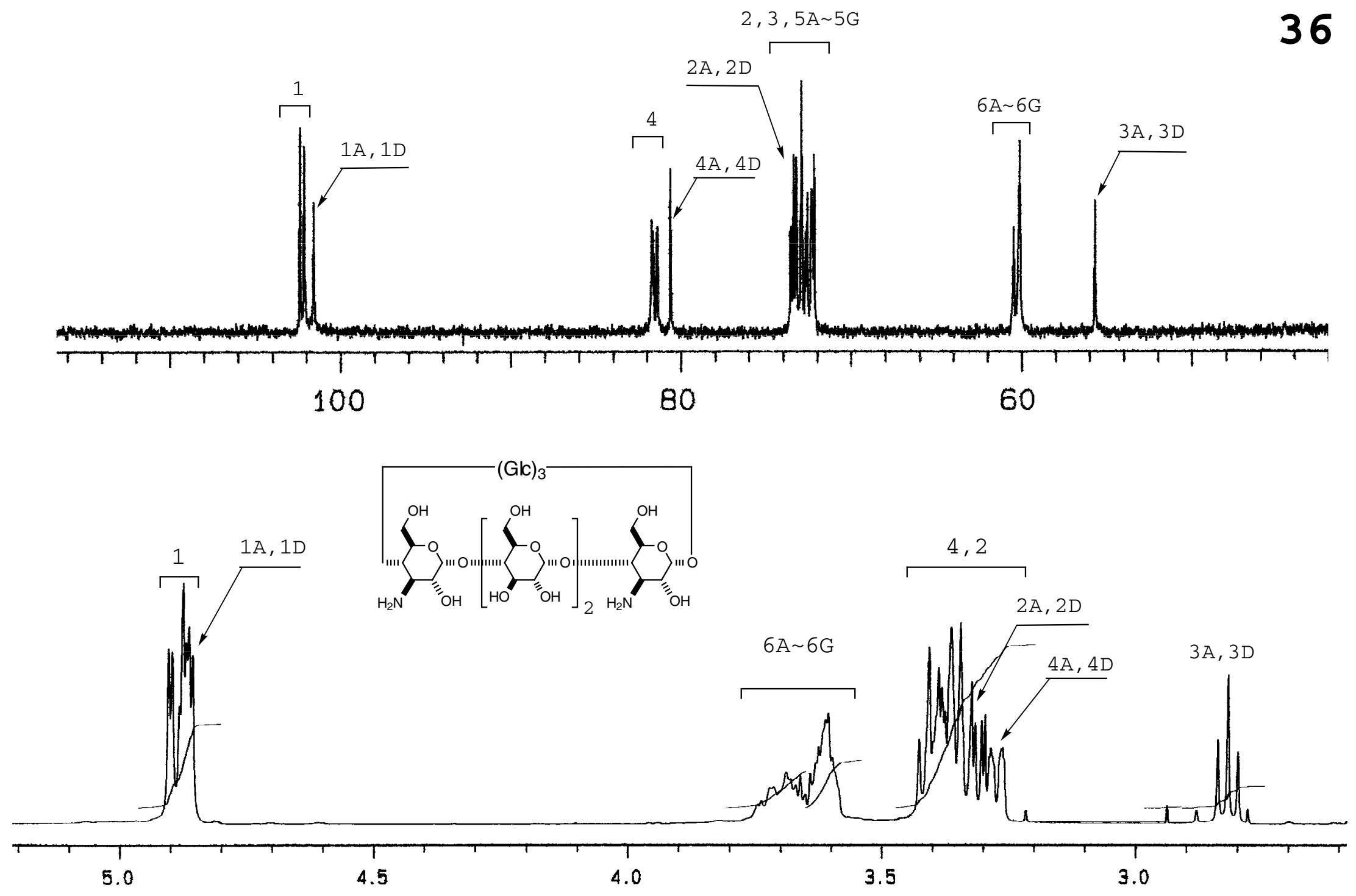

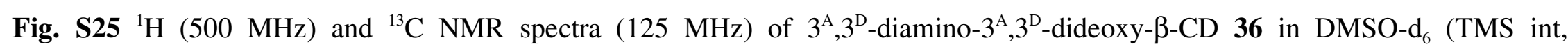
deuterated). 


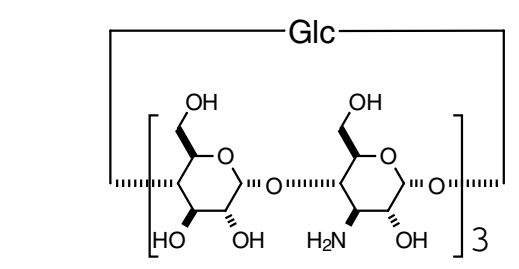

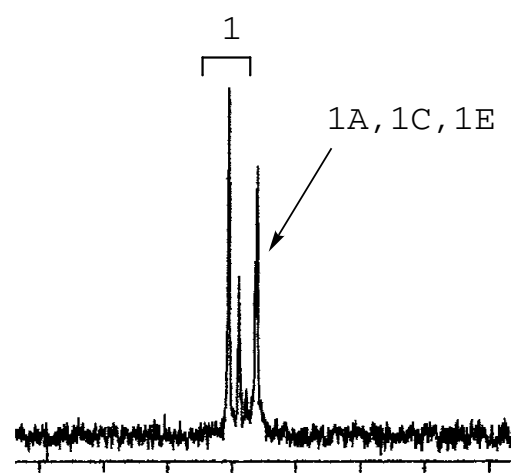

100

80

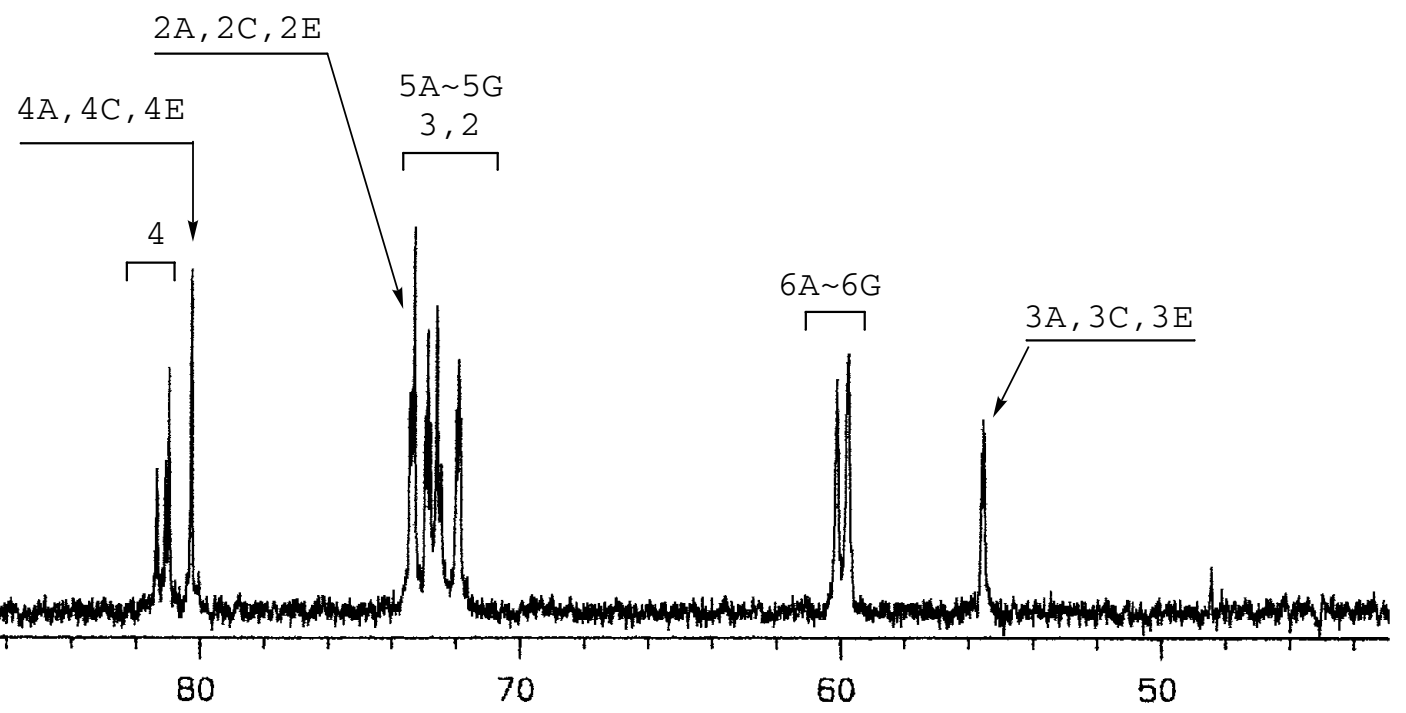

$6 \mathrm{~A} \sim 6 \mathrm{G}, 5 \mathrm{~A} \sim 5 \mathrm{G}, 3$

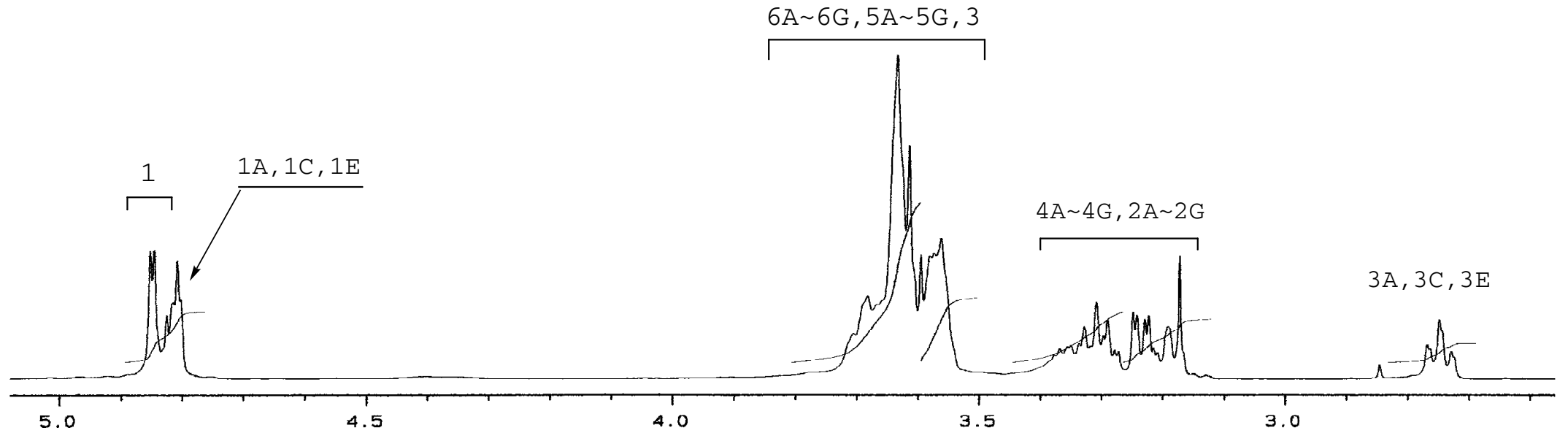

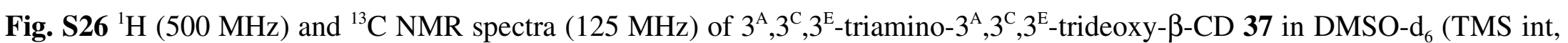
deuterated). 


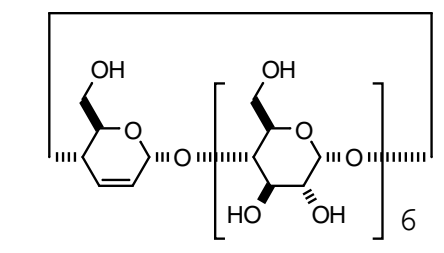

42
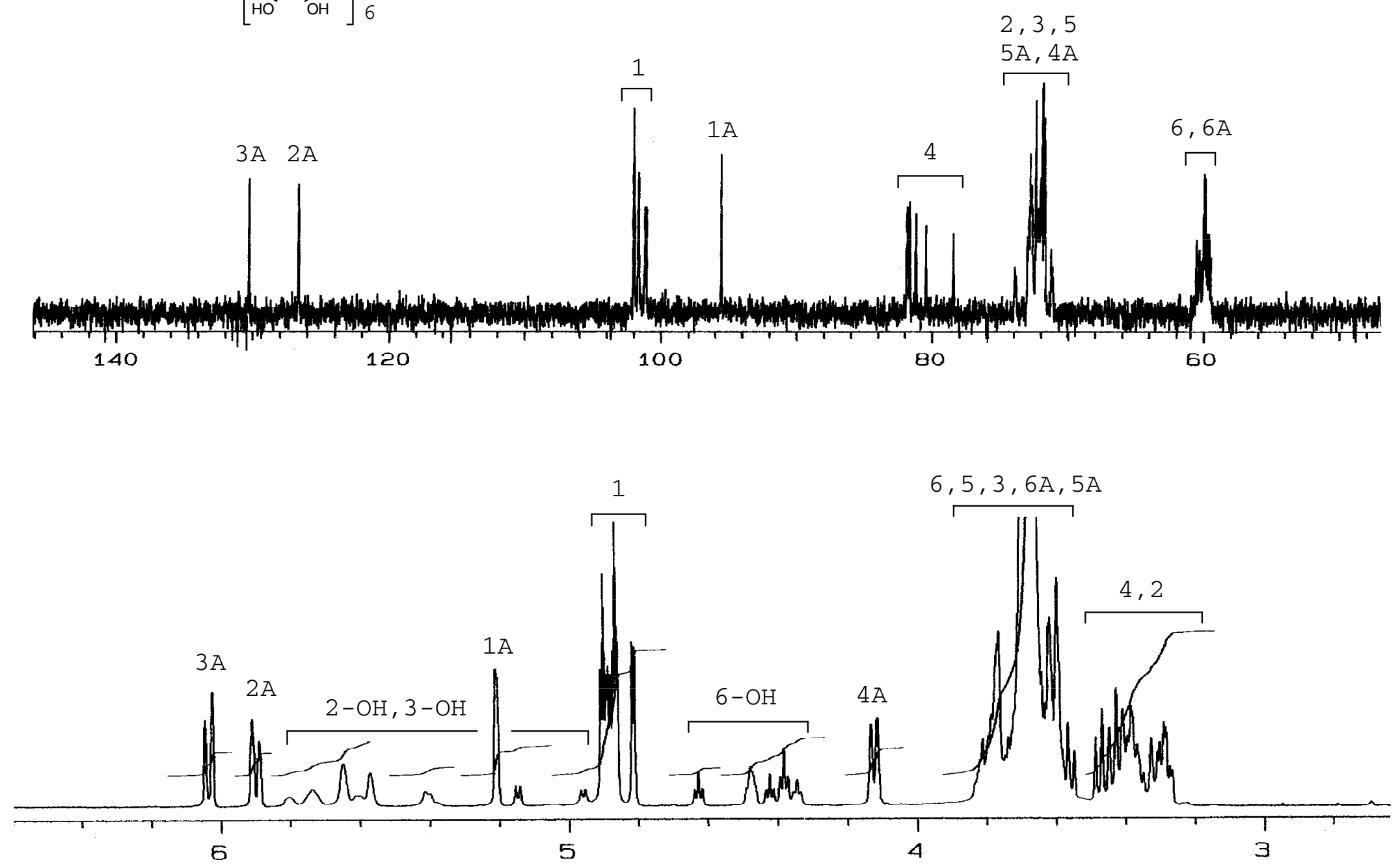

Fig. $\mathbf{S 2 7}{ }^{1} \mathrm{H}(500 \mathrm{MHz})$ and ${ }^{13} \mathrm{C}$ NMR spectra $(125 \mathrm{MHz})$ of $2^{\mathrm{A}}, 3^{\mathrm{A}}$-didehydro- $2^{\mathrm{A}}, 3^{\mathrm{A}}$-dideoxy- $\beta$-CD 42 in DMSO-d $\mathrm{d}_{6}(\mathrm{TMS}$ int). 


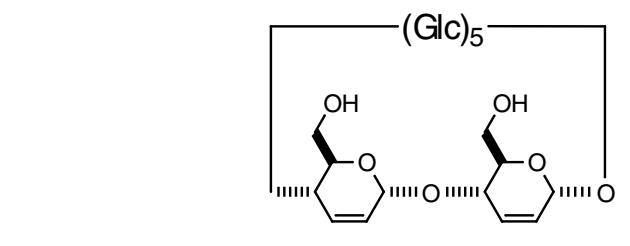

43
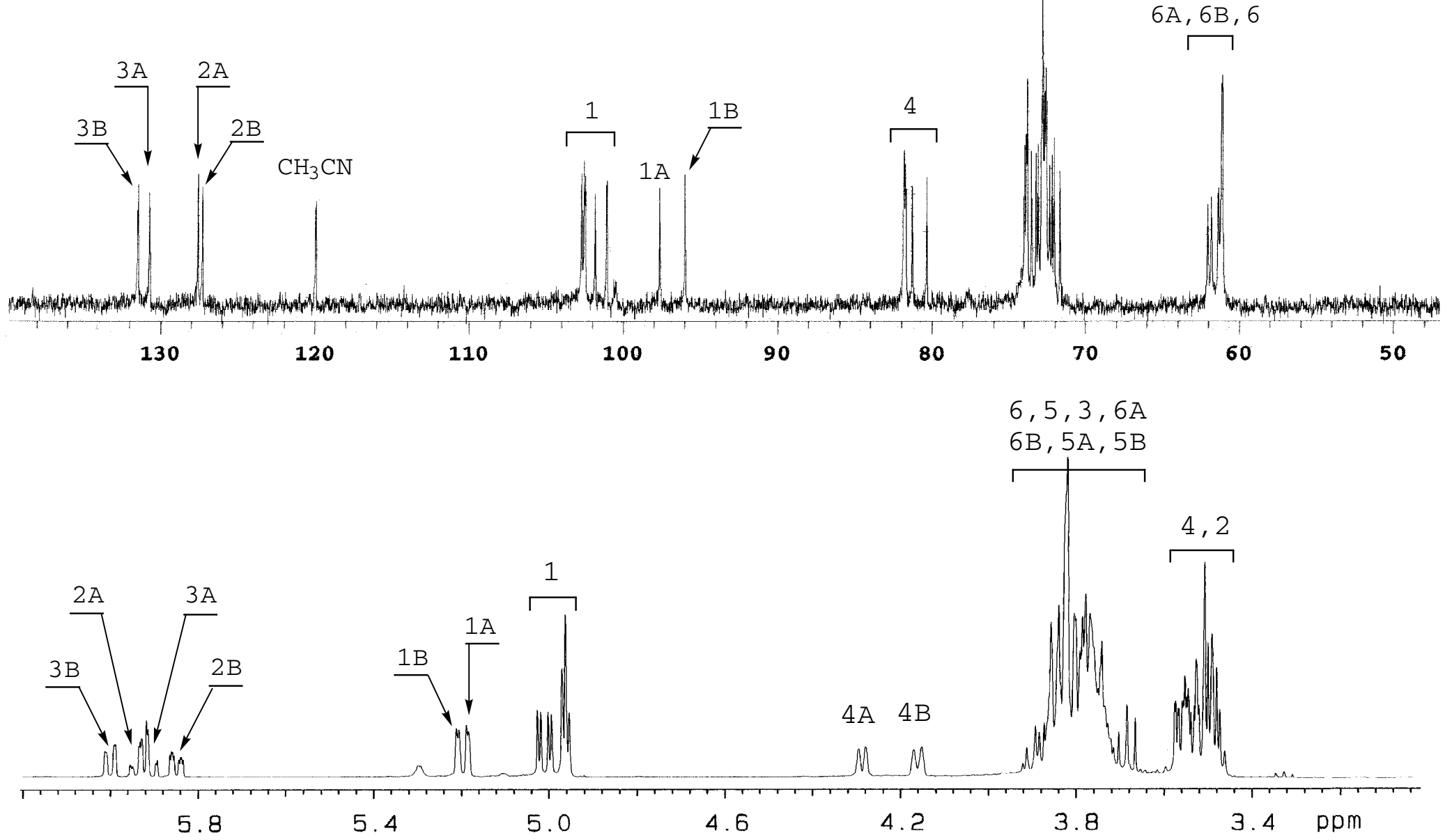

Fig. S28 ${ }^{1} \mathrm{H}(500 \mathrm{MHz})$ and ${ }^{13} \mathrm{C}$ NMR spectra $(125 \mathrm{MHz})$ of $2^{\mathrm{A}}, 2^{\mathrm{B}}, 3^{\mathrm{A}}, 3^{\mathrm{B}}$-tetradehydro- $2^{\mathrm{A}}, 2^{\mathrm{B}}, 3^{\mathrm{A}}, 3^{\mathrm{B}}$-tetradeoxy- $\beta$-CD 43 in $\mathrm{D}_{2} \mathrm{O}$ $\left(\mathrm{CH}_{3} \mathrm{CN}\right.$ int $)$. 


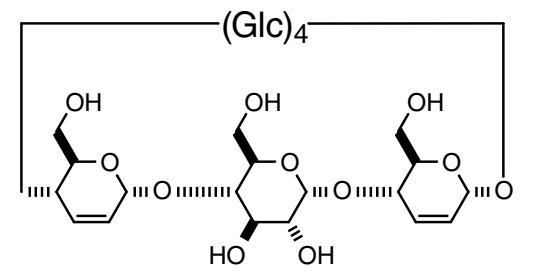

44
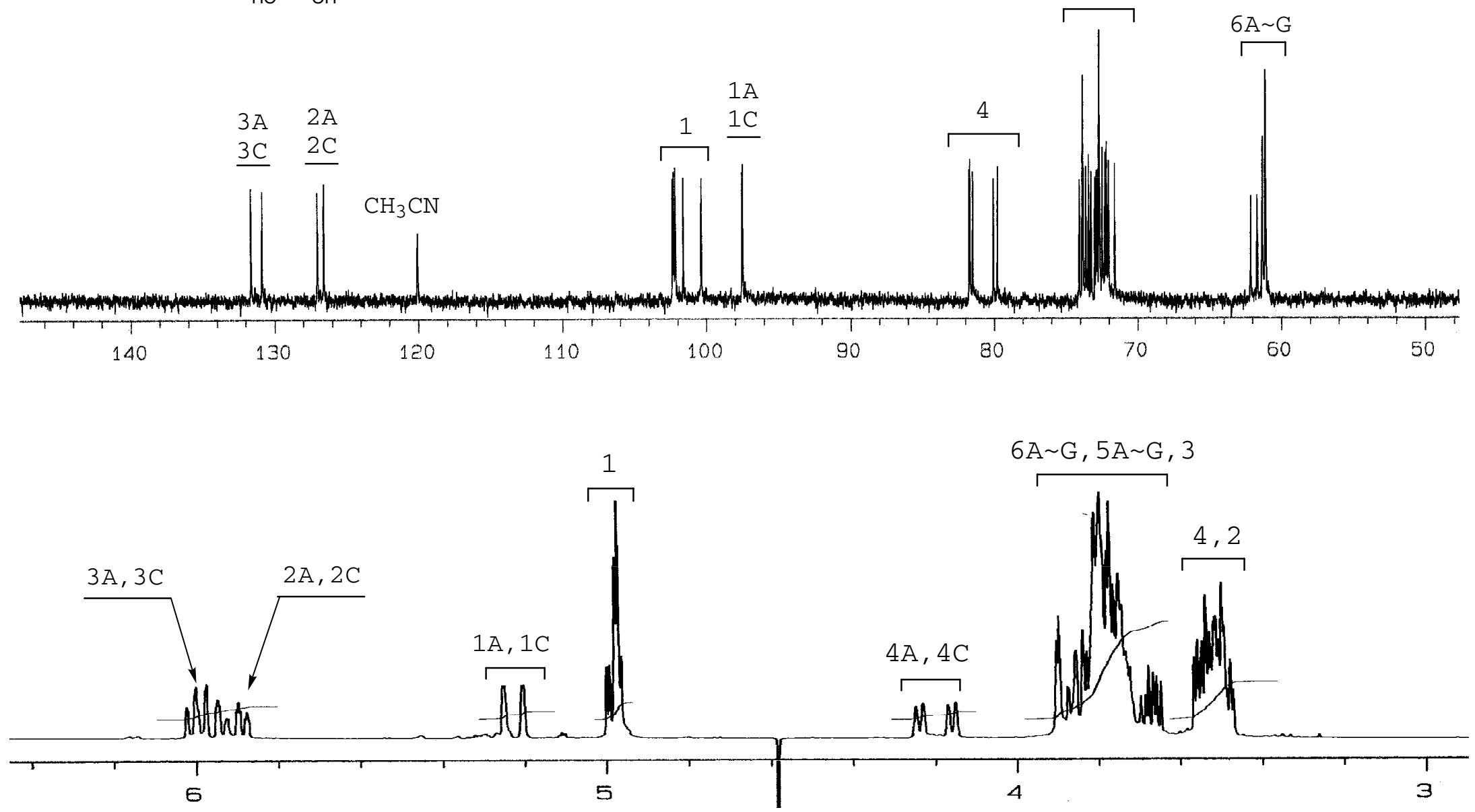

Fig. S29 ${ }^{1} \mathrm{H}(500 \mathrm{MHz})$ and ${ }^{13} \mathrm{C}$ NMR spectra $(125 \mathrm{MHz})$ of $2^{\mathrm{A}}, 2^{\mathrm{C}}, 3^{\mathrm{A}}, 3^{\mathrm{C}}$-tetradehydro- $2^{\mathrm{A}}, 2^{\mathrm{C}}, 3^{\mathrm{A}}, 3^{\mathrm{C}}$-tetradeoxy- $\beta$-CD 44 in $\mathrm{D}_{2} \mathrm{O}$ $\left(\mathrm{CH}_{3} \mathrm{CN}\right.$ int). 


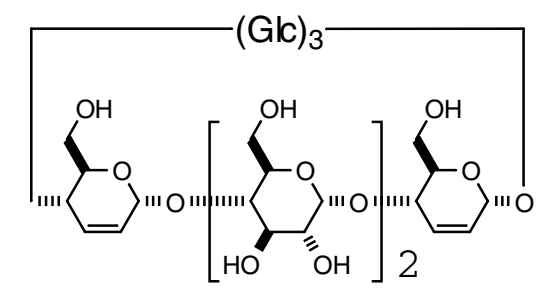

\section{5}
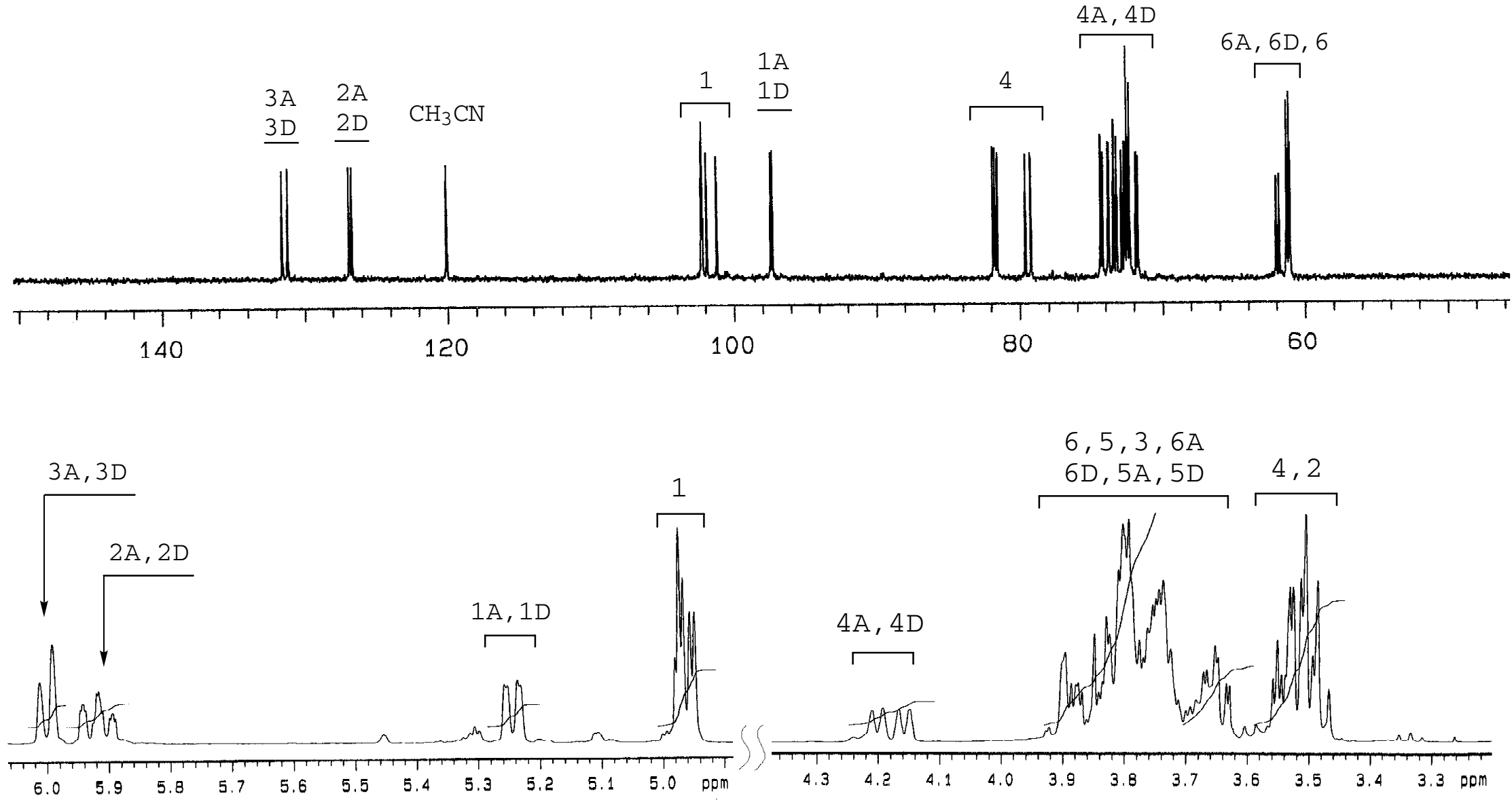

Fig. S30 ${ }^{1} \mathrm{H}(500 \mathrm{MHz})$ and ${ }^{13} \mathrm{C}$ NMR spectra $(125 \mathrm{MHz})$ of $2^{\mathrm{A}}, 2^{\mathrm{D}}, 3^{\mathrm{A}}, 3^{\mathrm{D}}$-tetradehydro- $2^{\mathrm{A}}, 2^{\mathrm{D}}, 3^{\mathrm{A}}, 3^{\mathrm{D}}$-tetradeoxy- $\beta$-CD 45 in $\mathrm{D}_{2} \mathrm{O}$ $\left(\mathrm{CH}_{3} \mathrm{CN}\right.$ int). 


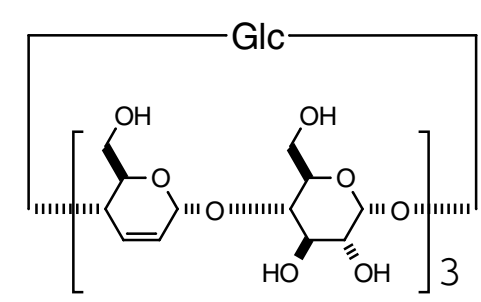

46

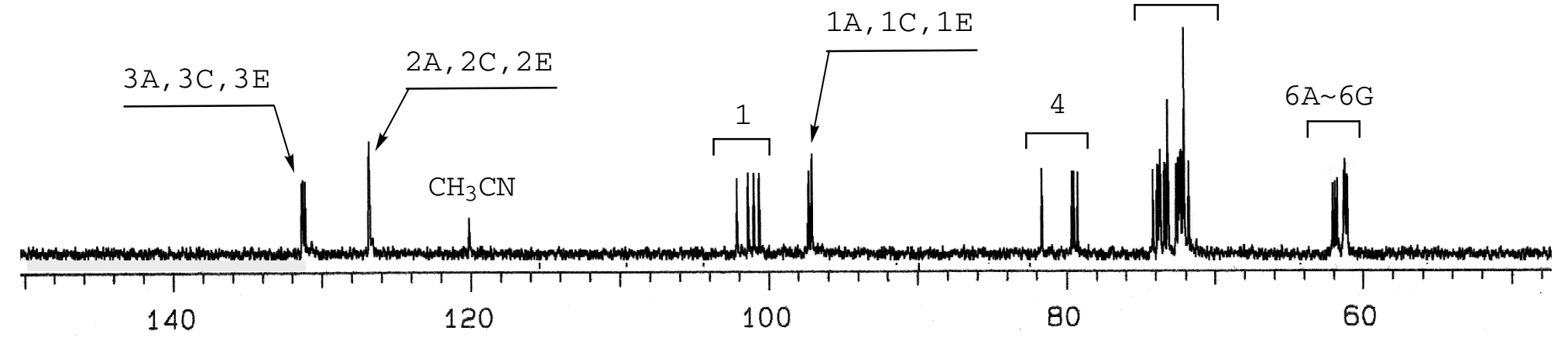

$6 A \sim 6 G$

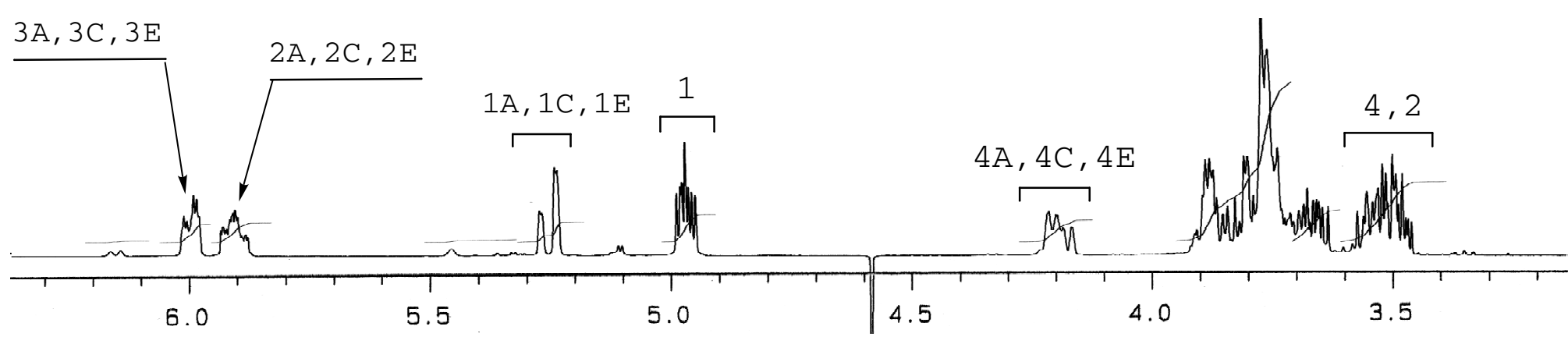

Fig. S31 ${ }^{1} \mathrm{H}(500 \mathrm{MHz})$ and ${ }^{13} \mathrm{C}$ NMR spectra $(125 \mathrm{MHz})$ of $2^{\mathrm{A}}, 2^{\mathrm{C}}, 2^{\mathrm{E}}, 3^{\mathrm{A}}, 3^{\mathrm{C}}, 3^{\mathrm{E}}$-hexadehydro- $2^{\mathrm{A}}, 2^{\mathrm{C}}, 2^{\mathrm{E}}, 3^{\mathrm{A}}, 3^{\mathrm{C}}, 3^{\mathrm{E}}$-hexadeoxy- $\beta$-CD 46 in $\mathrm{D}_{2} \mathrm{O}\left(\mathrm{CH}_{3} \mathrm{CN}\right.$ int). 


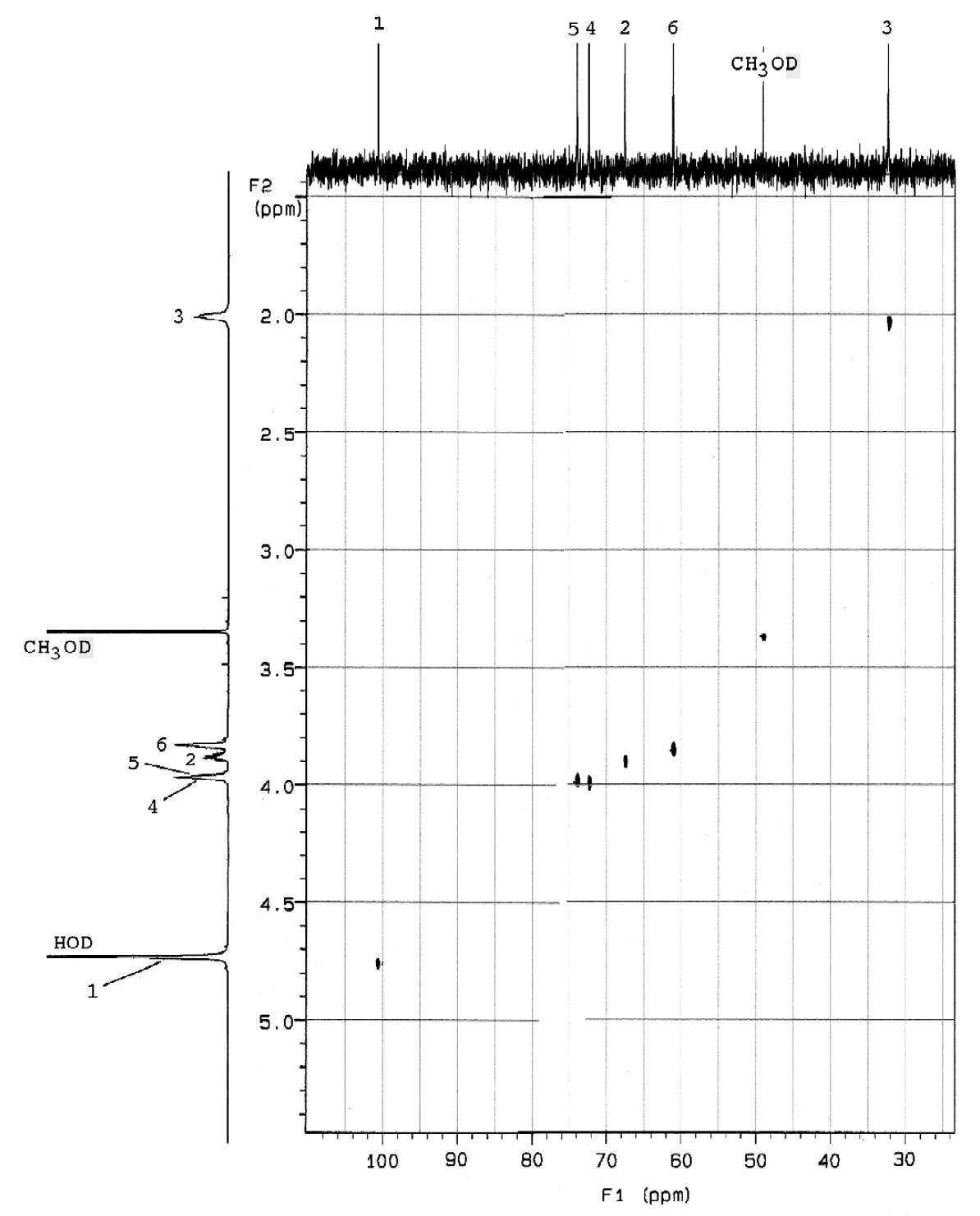

48

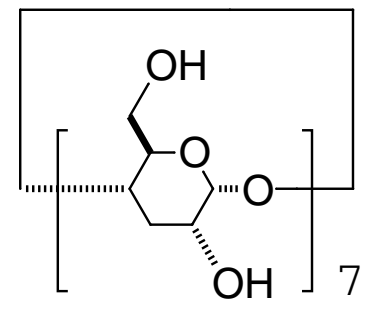

Fig. S32 ${ }^{1} \mathrm{H}_{-}{ }^{13} \mathrm{C}$ COSY NMR spectrum $(500 \mathrm{MHz})$ of per(3-deoxy)- $\beta$-cyclomannin 48 in $\mathrm{D}_{2} \mathrm{O}\left(\mathrm{CH}_{3} \mathrm{CN}\right.$ int $)$. 